\title{
Investigation of Archiving Techniques for Evolutionary Multi-objective Optimizers
}

\author{
Investigações de Técnicas de Arquivamento em Otimizadores Evolucionários \\ Multiobjetivo
}

Hudson Geovane de Medeiros ${ }^{1 *}$, Elizabeth Ferreira Gouvêa Goldbarg², Marco Cesar Goldbarg²

\begin{abstract}
The optimization of multi-objective problems from the Pareto dominance viewpoint can lead to huge sets of incomparable solutions. Many heuristic techniques proposed to these problems have to deal with approximation sets that can be limited or not. Usually, a new solution generated by a heuristic is compared with other archived non-dominated solutions generated previously. Many techniques deal with limited size archives, since comparisons within unlimited archives may require significant computational effort. To maintain limited archives, solutions need to be discarded. Several techniques were proposed to deal with the problem of deciding which solutions remain in the archive and which are discarded. Previous investigations showed that those techniques might not prevent deterioration of the archives. In this study, we propose to store discarded solutions in a secondary archive and, periodically, recycle them, bringing them back to the optimization process. Three recycling techniques were investigated for three known methods. The datasets for the experiments consisted of 91 instances of discrete and continuous problems with 2, 3 and 4 objectives. The results showed that the recycling method can benefit the tested optimizers on many problem classes.
\end{abstract}

Keywords: Archiving techniques - multi-objective evolutionary algorithms - recycling techniques.

Resumo: A otimização de problemas com múltiplos objetivos sob o ponto de vista da dominância de Pareto pode levar a um conjunto de soluções incomparáveis grande. Muitas técnicas heurísticas propostas para tais problemas precisam lidar com conjuntos de aproximação que podem ser limitados ou não. Geralmente, uma nova solução gerada por uma heurística é comparada com outras soluções arquivadas previamente. Muitas técnicas trabalham com conjuntos de tamanho limitado, uma vez que comparações com arquivos ilimitados podem demandar muito esforço computacional. Para manter arquivos de tamanho limitado, algumas soluções devem ser descartadas. Diversas técnicas foram propostas para lidar com o problema de decidir quais soluções devem ser mantidas ou descartadas. Estudos anteriores mostram que essas técnicas podem não previnir a deterioração dos arquivos. Neste trabalho, nós propomos o uso de um arquivo secundário para manter as soluções descartadas e, periodicamente, reciclá-las, trazendo-as de volta para o processo de otimização. Três técnicas de reciclagem são investigadas em três algoritmos conhecidos. O conjunto de dados utilizados nos experimentos consiste de 91 instâncias de problemas discretos e contínuos com 2, 3 e 4 objetivos. Os resultados mostraram que as técnicas de reciclagem podem beneficiar os algoritmos de otimização em várias classes de problemas.

Palavras-Chave: Técnicas de arquivamento - algoritmos evolucionários multiobjetivo - técnicas de reciclagem.

${ }_{1}^{1}$ Programa de Pós-graduação em Sistemas e Computação, UFRN

${ }^{2}$ Departamento de Informática e Matemática Aplicada, UFRN

*Corresponding author: hudsongeovane@gmail.com

DOI: https://doi.org/10.22456/2175-2745.80478 • Received: 20/02/2018 • Accepted: 06/09/2018

CC BY-NC-ND 4.0 - This work is licensed under a Creative Commons Attribution-NonCommercial-NoDerivatives 4.0 International License. 


\section{Introduction}

Multi-objective optimization problems are an important field of Computer Science, once real problems often have more than one objective. Compared to single objective problems, the decision-making with multiple objectives becomes more challenging. Given that solutions may be better for some objectives and worse on others, usually, there is not one optimal solution for multi-objective problems, but a set of them. Due to this fact, heuristic algorithms for multi-objective problems produce a set of solutions, which is an approximation of the optimal set. During optimization, most heuristic multiobjective algorithms keep a population or an archive of solutions that approximate the Pareto optimal set. Many important multi-objective optimizers use an external set of solutions to approximate this optimal set $[1,2,3]$.

Since the comparison of solutions within an unlimited size archive may require significant processing times, many multiobjective optimizers deal with limited size archives. To keep an archive bounded, it may be necessary to discard solutions. Several techniques were proposed to deal with the problem of deciding which solutions remain in the archive and which are discarded. They are called filtering techniques and some examples are: adaptive grid archive [4], hypervolume based archive [5], adaptive $\varepsilon$-approximate algorithm [6] and SPEA2 [1], among others. Knowles and Corne [7] present a review about bounded archiving. Other studies showed that the filtering technique impacts on the quality of the final approximation set produced by the optimizer. The analysis presented by [8] revealed that most archiving techniques could lead to the deterioration of the archive. This occurs when, along the optimization process, the approximation set maintained by the optimizer keeps a solution that is worse than a previously discarded one, and sometimes allows an optimizer storing an entire set worse than another from a previous iteration.

To soften the deterioration problem, we propose to recycle discarded solutions bringing them back to the optimization process. We investigate three recycling methods which were tested with three evolutionary algorithms proposed for multiobjective problems: NSGAII, SPEA2, and PAES. These algorithms were chosen because they are widely used in the literature, and their archiving techniques were analyzed previously on [8] and [9]. These optimizers and their versions implemented with the recycling techniques are applied to discrete and continuous problems.

This study also extends the analysis presented by [8] with the inclusion of a theoretical and empirical investigation of the properties of four archiving techniques not investigated in that work. The filtering techniques analyzed are Non-dominated Sorting Genetic Algorithm-II [10], Strength Pareto Evolutionary Algorithm 2 [1], Adaptive Grid Archive [4], Multi-level Grid Archive [11], Ideal Archive, Distributed Archive, Distance Archive [12] and Adaptive Rectangle Archive [13].

The remainder of this paper is organized as follows. Basic concepts of multi-objective optimization problems and algorithms are presented in Section 2. Section 3 presents some existing archiving techniques and extends the results presented by [8] with the inclusion of four archiving techniques. Section 4 presents a computational experiment with 10 archiving techniques and 7 input sequences considering 2 , 3 and 4 objectives. The recycling methods are presented in Section 5. The results of a computational experiment based on 91 instances of discrete and continuous problems are presented in Section 6. Finally, some conclusions are presented in Section 7.

\section{Basic Concepts}

A general multi-objective minimization problem can be defined as in equation 1, where $x \in X$ is a solution, $X \subseteq \mathfrak{R}^{n}$ is the set of feasible solutions, $f: \Re^{n} \rightarrow \Re^{d}$ is the function that maps $x$ to its objective vector $f(x), n$ and $d$ are integers. $Y$ denotes the image of $X$ in the objective space, $y=f(x), y \in Y$.

$$
\min _{x \in X} f(x)=\left(f_{1}(x), \ldots, f_{d}(x)\right)
$$

Since a solution of a multi-objective problem is mapped to a vector in the objective space, and not to a real number, the comparison of solutions in a multi-objective scenario is more complex than it is for single objective problems. Binary relations are used to define a partial order over the objective space. Some of those relations are presented in definitions $1-3$ and optimal solutions are defined in 4 .

Definition 1 y dominates $y^{\prime}$, denoted by $y \prec y^{\prime}$, iff $f_{i}(x) \leq$ $f_{i}\left(x^{\prime}\right), i=1, \ldots, d$, and $\exists k, 1 \leq k \leq d$ such that $f_{k}(x)<f_{k}\left(x^{\prime}\right)$. Accordingly, $x$ dominates $x^{\prime}$.

Definition 2 y weakly dominates $y^{\prime}$, denoted by $y \preceq y^{\prime}$, iff $f_{i}(x) \leq f_{i}\left(x^{\prime}\right), i=1, \ldots, d$. Accordingly, $x$ weakly dominates $x^{\prime}$.

Definition 3 y is incomparable to $y^{\prime}$, denoted by $y \| y^{\prime}$, if neither $y \prec y^{\prime}$ nor $y^{\prime} \prec y$. Accordingly, $x$ is said to be incomparable to $x^{\prime}$.

Definition 4 A solution $x^{*} \in X$ is called efficient or Pareto optimal iff $\nexists x \in X$ such that $f(x) \prec f\left(x^{*}\right)$.

The set of optimal solutions is called efficient set or Pareto optimal set and is denoted by $X^{*}$. The image of $X^{*}$ in the objective space is denoted by $Y^{*}$ and is called Pareto optimal front.

Many heuristics for multi-objective optimization problems focus on delivering sets of objective vectors that are mutually incomparable. A set of mutually incomparable objective vectors is said to be a nondominated set. Those sets are called approximation sets. Sets of objective vectors can also be investigated from a dominance perspective as in definition 5 .

Definition 5 Let $A$ and $B$ be two sets of objective vectors, $A \neq B$. If every $b \in B$ is weakly dominated by at least one element $a \in A$, then $A$ is said to be better than $B$, denoted by $A \triangleright B$. 
The $\triangleright$ order relation establishes that $A$ is better than $B$ if and only if $A$ dominates the whole area of the objective space dominated by $B$. When two nondominated sets are compared, however, the $\triangleright$ relation can be not sufficient. For instance, situations in which the sets assessed have exclusive dominating areas, i.e., areas of the objective space that are dominated only by one of them. Let $A$ and $B$ be two different nondominated sets, such that there are areas of the objective space exclusively dominated by $A$ and others by $B$. Then, neither $A \subset Y^{*}$ nor $B \subset Y^{*}$. In those and other situations, other comparison methods need to be adopted to improve the decision-making.

In this study, an algorithm for a multi-objective optimization problem is called optimizer. Some optimizers' designers opt to keep a limited size archive to store nondominated solutions generated during the search process. This option is made once a huge number of nondominated solutions can be generated and their maintenance in a single archive can be computationally expensive. When the number of incomparable solutions grows, the computational effort to compare a newly generated solution to those stored in the archive becomes significant when compared to other operations. The limited size archive can be the population of solutions managed by the algorithm or an external archive which works as a repository. An advantage of using a limited size archive is that less computational effort is necessary to its maintenance. A disadvantage is that "good" solutions may be lost. During the optimization process, a huge number of solutions are generated and evaluated, and as the archive is limited, many of them are discarded, even if they are not dominated by any other solution generated so far. It is desirable that the limited size archive maintains a good approximation set, according to some criteria. Let $N$ be the maximum size of a limited archive, an optimal approximation set of bounded size $N$ is explained in Definition 6.

Definition 6 Let $A$ be a set of objective vectors and $|A| \leq N$, $N$ integer. $P$ is called an optimal approximation set of bounded size $N$ of $Y^{*}$ if there exists no set $A^{\prime} \subseteq Y$, such that $\left|A^{\prime}\right| \leq N$ and $A^{\prime} \triangleright A$.

In the remainder of this work, the limited size archive combined with the strategy used to its maintenance is called archiver. A seminal paper on archiving techniques was presented by [8].

\section{Archivers}

Algorithm 1 shows the general template of an archiver [8], $A$, limited to $N$ solutions. $A_{t-1}$ denotes the content of $A$ at the $t-1$-th iteration and $y$ a new solution to be inserted in $A$. If $y$ is dominated by at least one solution of $A_{t-1}$ (Line 1), then $y$ is discarded. Similarly, if $y$ dominates at least one solution of $A_{t-1}, y$ is stored and the solutions dominated by $y$ are removed. In case $y$ is incomparable to each solution of $A_{t-1}$ and $\left|A_{t-1}\right|<N, y$ is stored. The problem occurs when $y$ is incomparable to each solution of $A_{t-1}$ and $\left|A_{t-1}\right|=N$. In this case, a decision has to be made between to discard $y$ or store it and discard another solution from $A_{t-1}$. The maintenance strategy is implemented in filter () which defines whether $y$ is discarded or not and, in the last case, which solution of $A_{t-1}$ is removed. The implementation of filter () defines different archiving techniques. The $n d s()$ function returns a set of non-dominated solutions.

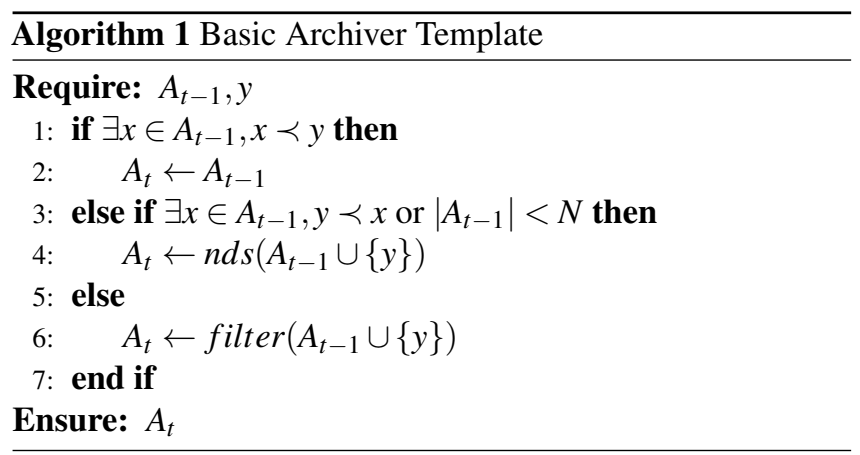

Many techniques for filter() were proposed in the literature. They were firstly analyzed by [8] and later by [9]. These analyses were concerned about desirable properties of the archivers and experimental results regarding quality indicators. The investigated properties, as presented by [8], are revised in Section 3.1 and the archivers in Section 3.2.

\subsection{Properties and Convergence}

For the sake of simplification, solutions are referred as objective vectors or merely as points.

Property 1 Diversifies. An archiver is said to be efficiency preserving when during updating $A_{t}$ to $A_{t+1},\left|A_{t}\right|=N$, only points of the dominating region of $A_{t}$ are allowed to be accepted to $A_{t+1}$. If an archiver does not have this property, then it is said to diversify [14].

Property 2 Monotone. An archiver is called monotone if there exists no possibility to one solution from $A_{t}$ dominates any solution from $A_{u}$ if $u>t . \forall x \in A_{u}, \forall x^{\prime} \in A_{t}, x \prec x^{\prime} \Rightarrow u>t$.

Property $3 \triangleright$-Monotone. An archiver is said $\triangleleft$-monotone if and only if $\forall A_{t}, A_{u} \subset \Omega, A_{t} \triangleright A_{u} \Rightarrow t>u$. This property is similar to monotone, but with $\triangleright$ relation between sets, not solutions. Obviously, if any archiver is not $\triangleright$-monotone, it will not be monotone.

Property $4 \subseteq Y^{*}$. An archiver has this property if it contains only optimal solutions from the processed input sequence, i.e., $\forall t, A_{t} \subseteq Y_{t}^{*}$.

Property 5 Limit-stable. Let $P$ be a set of solutions, and X an archiver. If inserting indefinitely points from $P$ into $X$ makes $X$ to converge to a stable set $X_{t}$ in finite time, then $X$ has the limit-stable property. 
Property 6 Limit-optimal. Let $P$ be any set of solutions, and $X$ an archiver. If inserting indefinitely points from $P$ into $X$ makes $X$ converges to an optimal approximation set (see Definition 6) $X_{t}$ in finite time, then $X$ has the limit-optimal property.

\subsection{Methods and Selection Algorithms}

Unbounded Archiver. The archive is not bounded. Given an input sequence of points, the unbounded archiver leads to the Pareto Front of this sequence.

Dominating Archiver. In this technique, a new point $y$ is accepted only if $\left|A_{t-1}\right|<N$ or if $y$ dominates at least one solution of $A_{t-1}$. Its filter $\left(A_{t-1} \cup\{y\}\right)$ function returns $A_{t-1}$. Although this archiver does not diversify, it does not ensure the $\subseteq Y^{*}$ property.

NSGA-II. The archiving technique named NSGA-II in this study comes from the selection mechanism of the multiobjective genetic algorithm proposed by [10]. Since the population of that genetic algorithm is limited, the selection mechanism defines which solutions go to the next generation. Here, the filter procedure consists in discarding the point from $\{y\} \cup A_{t-1}$ in the most crowded region. To measure how much crowd is the region of a point, the crowding distance is computed. The method to compute the crowding distance is presented in Algorithm 2. The list of solutions is sorted by the first objective, then by the second and so on. The crowding distance of the $i$-th solution is computed from the distance from $i$ to the immediately previous $(i-1)$ and next $(i+1)$ solutions. The solution with the smallest crowding distance is discarded. As shown by [8], the NSGAII archiving technique cannot guarantee the convergence of the archiver to an optimal non-dominated set. The archiver can deteriorate and $\triangleleft$-deteriorate (it is not monotone nor $\triangleleft$-monotone).

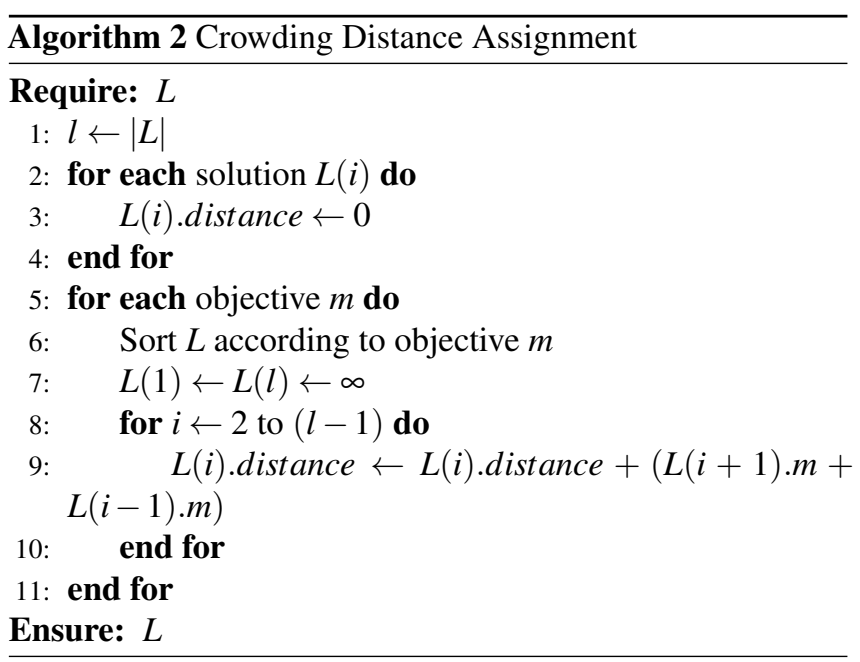

SPEA2. The archiving technique named SPEA2 also comes from a genetic algorithm proposed for multi-objective problems presented by [1]. In this case, the filter () function calculates first the distance between every pair of points in $A$, associates the minimum distance computed for each point with that point and discards the point associated with the overall minimum distance. Ties are broken by considering the second smallest distance, and so on. As the NSGAII archiver, its content may $\triangleleft$-deteriorate [8].

Adaptive Grid Archiver (AGA). This archiver was proposed by [5]. The objective vectors are split into a grid. Each cell of the grid may contain many solutions. filter() removes one solution, chosen at random, from the most crowded cell, excluding a minimum or maximum on any objective. It is not monotone nor $\triangleleft$-monotone [8].

Hypervolume Archiver (AA). This archiver technique was proposed by [5]. It aims at maximizing the hypervolume of the approximation set. Its filter() function returns the subset of $A_{t-1} \cup\{y\}$, limited to size $N$, with the maximum hypervolume. As the hypervolume calculation is exponential on the number of objectives, AA may spend more time than the other techniques. Besides, this greedy approach does not ensure that the hypervolume will be maximum at the end of the input sequence, as shown by [15].

Multi-level Grid Archiving (MGA). Proposed by [11], the MGA uses a hierarchical family of boxes of different levels over the objective space. Its filter() function finds the smallest level $b$ where at least one solution from the boxes at level $b$ is dominated. If the point to be inserted is one of the dominated points at level $b$, then it is rejected. Otherwise, $a$ random point is removed. MGA can deteriorate, as shown by [8], but it is $\triangleleft$-monotone.

Ideal Archiver (Ideal). This archiver was proposed by [12] to control convergence in particle swarm optimization. It first calculates an ideal point, which is the vector with the best values for each objective. After that, the archiver discards the point with higher Euclidean distance from the ideal point. The main idea behind this technique is to converge to only one area of the objective space.

Distributed Archiver (Distributed). This archiver also uses the ideal point to guide the filter() function. However, its convergence is not directed to only one point, but to $d+$ 1 points - the ideal point and one reference point for each dimension. The reference points are the closest points to each dimension, defining $d+1$ regions of the objective space (one next to ideal point and $d$ to each reference point). Each region stores $(N+1) /(D+1)$ points, i.e., the number of points divided by the number of regions. For each dimension, each set consists of the $(N+1) /(D+1)$ closest points to each reference point. The point farthest from any reference point is discarded and $A_{t}$ is defined as the union of the reference sets. This scheme does not guarantee the archiver to be $\triangleleft$ monotone, and consequently, it is not monotone and has not the $\subseteq Y^{*}$ property.

Distance to Reference Points Archiver (Distance). Also proposed by [12], it works similarly to the Distributed Archiver, first calculating the same reference points. However, it does not limit the region size to $(N+1) /(D+1)$. For each point in $A_{t-1} \cup\{y\}$ the smaller distance from each reference point is calculated. The point with the higher value is discarded. 
Distance Archiver only has the Diversify property, out of the six presented before.

Adaptive Rectangle Archiver (ARA). This one does not follow the pattern of Algorithm 1 and it is not limited to $N$ points. ARA is based on two steps: to define a region adaptively and divide it into rectangles, such that each rectangle only contains one point [13]. The region is defined by two vectors $a^{(m i n)}$, which is the same Ideal Point proposed by [12], and $a^{(\max )}$, the vector with the worst objective values for each dimension in $A$. ARA uses the E-dominance concept to decide in which rectangle a point is placed. E-dominance concept is similar to $\varepsilon$-dominance, except from $E \in \mathfrak{R}^{d}$, while $\varepsilon \in \mathfrak{R}$. So, the lowest the value of $E_{i}$, more finely and accurately the dimension of the $i$-th objective is split. Moreover, the archiver saves the same reference points of the Distributed Archiver, keeping a set of $d$ solutions with the best values of each objective found so far. The archiver has the six desirable properties. However, due to its flexible structure, it has some problems that are presented in the next section.

Table 1 shows a summary of the properties of each archiver and extends the table presented by [8]. Whereas most of the archivers are not monotone, and only two of them have the $\subseteq Y^{*}$ property (one is the unbounded archiver), the main idea of this work emerges: to recycle discarded solutions and bring them back to the optimization process.

Table 1. Archivers and their properties

\begin{tabular}{lcccccc}
\hline Archiver & Div. & Mon. & $\triangleleft-$ Mon. & $\subseteq Y^{*}$ & L-St & L-Op \\
\hline Unbounded & $\checkmark$ & $\checkmark$ & $\checkmark$ & $\checkmark$ & $\checkmark$ & $\checkmark$ \\
Dominating & $x$ & $\checkmark$ & $\checkmark$ & $x$ & $\checkmark$ & $\checkmark$ \\
NSGA-II & $\checkmark$ & $x$ & $x$ & $x$ & $x$ & $x$ \\
SPEA2 & $\checkmark$ & $x$ & $x$ & $x$ & $x$ & $x$ \\
AGA & $\checkmark$ & $x$ & $x$ & $x$ & $x$ & $x$ \\
AA & $\checkmark$ & $x$ & $\checkmark$ & $x$ & $\checkmark$ & $\checkmark$ \\
MGA & $\checkmark$ & $x$ & $\checkmark$ & $x$ & $\checkmark$ & $\checkmark$ \\
Ideal & $\checkmark$ & $x$ & $\checkmark$ & $x$ & $\checkmark$ & $\checkmark$ \\
Distributed & $\checkmark$ & $x$ & $x$ & $x$ & $x$ & $x$ \\
Distance & $\checkmark$ & $x$ & $x$ & $x$ & $x$ & $x$ \\
ARA & $\checkmark$ & $\checkmark$ & $\checkmark$ & $\checkmark$ & $\checkmark$ & $\checkmark$ \\
\hline
\end{tabular}

\section{Experiments with Fixed Input Se- quences of Points}

We analyzed the quality of the sets generated by each archiver presented in Section 3.2 for fixed input sequences. We tested each archiver with different input files, each of them consisting of a set of points (objective vectors). The experiment consisted in adding each point of the input file iteratively to a limited size archive, initially empty. The quality of the set stored by each technique was assessed according to the quality indicators presented in Section 2 and the number of optimal solutions stored at the end of the process. This experiment extended the one reported by [8] with the inclusion of 3 new input files and 4 archivers. The new input files are different from those presented by [8] with regard to dimension, scale and structure. They were created to investigate specific problems of the archivers proposed by [12] and [13]. The value of each component of vector $E$, used by ARA, was $E_{i}=\pi / 100, \forall i=$ $1, \ldots, d$.

The archivers were tested with 7 input files: (1) smallPF2d-10000, (2) smallPF-3d-10000, (3) 2000-3d, (4) 10000-4d, (5) clustered-2d-900, (6) 1to2-2d-2000 and (7) 1000-clustered$3 D$. Sequences $1,2,5$ and 6 were provided by [8].

Files smallPF-2d-10000 and smallPF-3d-10000 have 10,000 points with 2 and 3 objectives. Their optimal sets contain 970 and 1,335 points, respectively. They were generated from a real optimizer solving a problem. The 1 to $2-2 d-2000$ file contains a sequence of 2,000 incomparable points, from $(1,2)$ to $(2,1)$, with the first objective increasing while the second decreases. Files 2000-3d and 10000-4d contain 2,000 and 4,000 points with 3 and 4 objectives. They were created by a specific algorithm that generates random points with objective values between 0 and 1. 1000-clustered-3D and clustered-2d-900 are files containing, respectively, 1000 and 900 clustered points with 3 and 2 objectives. These files were used to investigate some properties of ARA. The archives were limited to $N=100$, except the one obtained with ARA, which is not limited due to the archiver's structure.

Table 2 shows the number of optimal solutions saved by each archiver. Line 1 shows the references to the files. Line Unbounded shows the size of the optimal set of the points contained in each file. Dominating and MGA finished the process with archives containing only optimal solutions for all input sequences. AA, Ideal, Distributed and Distance failed to find optimal solutions only in one case, losing 1, 2, 23 and 14 optimal solutions, respectively. The NSGA-II archiver presented a weak result on the 4-dimension file, which showed to be also the hardest instance for SPEA2, Ideal, Distributed and Distance. The average number of optimal points for each archiver were: 100 to Dominating and MGA, 99.8 for AA, 99.6 for Ideal, 97.2 for Distance, 96.2 for AGA, 95.4 for Distributed, 94.4 for SPEA2, 83.4 for NSGA-II and 45.4 for ARA.

Except for the 4-dimensional file, ARA managed to keep significantly fewer solutions than the other techniques. The results show that ARA could not keep a representative number of solutions when tested to two-dimensional vectors. Besides, when tested to clustered points, the archiver presented the same problem, even if the vectors had a higher dimension.

We applied the hypervolume [16], denoted $H Y P()$, and the unary additive $\varepsilon$ [17], denoted by $\varepsilon_{a d d}()$ to the final sets. Tables 3 and 4 show the results of the hypervolume and $\varepsilon$-add indicators, respectively. The hypervolume is maximized and the $\varepsilon$-add minimized. The results presented in table 3 are percentages of the Pareto Front hypervolume. The archivers were ranked according to average results and presented in this order. The values on Table 4 have to be multiplied by $10^{4}$ on files 1 and 2 , by $10^{2}$ on files 3,4 and 6 , and by $10^{3}$ on file 5 .

Although [15] showed that being greedy did not ensure that the hypervolume is maximum at the end of the input sequence, except from 1to2-2d-2000 (instance 6), AA presented 
Investigation of Archiving Techniques for Evolutionary Multi-objective Optimizers

Table 2. Number of optimal points stored by each archiver technique.

\begin{tabular}{lrrrrrrr}
\hline Archiver & 1 & 2 & 3 & 4 & 5 & 6 & 7 \\
\hline Unbounded & 970 & 1,335 & 500 & 1000 & 300 & 2,000 & 93 \\
Dominating & 100 & 100 & 100 & 100 & 100 & 100 & 93 \\
NSGA-II & 94 & 99 & 97 & 27 & 100 & 100 & 93 \\
SPEA2 & 94 & 99 & 99 & 80 & 100 & 100 & 93 \\
AGA & 91 & 95 & 100 & 95 & 100 & 100 & 93 \\
AA & 99 & 100 & 100 & 100 & 100 & 100 & 93 \\
MGA & 100 & 100 & 100 & 100 & 100 & 100 & 93 \\
Ideal & 100 & 100 & 100 & 98 & 100 & 100 & 93 \\
Distributed & 100 & 100 & 100 & 77 & 100 & 100 & 93 \\
Distance & 100 & 100 & 100 & 86 & 100 & 100 & 93 \\
ARA & 8 & 47 & 39 & 131 & 2 & 2 & 3 \\
\hline
\end{tabular}

Table 3. Hypervolume results for each archiver.

\begin{tabular}{lccccccc}
\hline Archiver & 1 & 2 & 3 & 4 & 5 & 6 & 7 \\
\hline Unbounded & 100 & 100 & 100 & 100 & 100 & 100 & 100 \\
AA & 99.99 & 99.99 & 99.98 & 99.92 & 99.97 & 99.79 & 100 \\
SPEA2 & 99.99 & 99.99 & 99.89 & 94.03 & 99.96 & 89.70 & 100 \\
NSGA-II & 99.99 & 99.99 & 99.44 & 77.17 & 99.94 & 99.85 & 100 \\
AGA & 99.99 & 99.99 & 99.45 & 97.70 & 99.43 & 99.38 & 100 \\
MGA & 99.97 & 99.85 & 99.95 & 99.39 & 99.90 & 99.38 & 100 \\
Dominating & 99.99 & 99.99 & 99.41 & 99.04 & 99.41 & 59.94 & 100 \\
ARA & 99.97 & 99.99 & 97.79 & 99.18 & 4.62 & 85.72 & 82.73 \\
Ideal & 98.97 & 98.80 & 99.97 & 99.79 & 93.07 & 59.94 & 100 \\
Distributed & 99.99 & 99.97 & 99.20 & 95.87 & 85.12 & 87.08 & 100 \\
Distance & 99.97 & 99.92 & 90.28 & 89.58 & 34.67 & 87.08 & 100 \\
\hline & & & & & & &
\end{tabular}

the best results for all instances. The Dominating archiver, obviously, kept the first 100 vectors until the end of the experiment, which resulted in a poor output, considering both quality indicators.

As AA verifies the best set (regarding hypervolume) each iteration, it is natural to present the best performance this indicator. The archivers proposed by [12] did not go well for this indicator, and they were ranked in the last positions. A conclusion is that concentrating the points is not a good strategy if one wishes to maximize the hypervolume, once this indicator aims at measuring how good is the distribution of the points of a set.

MGA and AA had the best $\varepsilon$ indicator results. Despite the fact that AA has higher time complexity than the other archivers, it can be considered the winner of these experiments for the two indicators. Although the MGA performed well for the $\varepsilon$-add, it was ranked in the 5th place for the previous indicator. Distance Archiver had poor results for this indicator, as well. Its strategy may lead the archive to $\triangleleft$-deteriorate easily, as shown by [9].

Table 5 presents the times spent by each archiving technique to process the input sequences. AA and ARA were the most time-consuming archivers, in particular for the fourdimensional instance, which indicates that if they were used within a heuristic algorithm, there would be an excessive spending to deal with archiving. Dominating archiver was the fastest one, as expected, once it is not allowed to diversify and just refuses non-dominated points when out of capacity.
Table 4. $\varepsilon$-add indicator results for each archiver.

\begin{tabular}{lccccccc}
\hline Archiver & 1 & 2 & 3 & 4 & 5 & 6 & 7 \\
\hline MGA & 0.60 & 4.40 & 0.65 & 5.25 & 1.85 & 1.55 & 0 \\
AA & 0.60 & 23.1 & 1.04 & 3.86 & 1.67 & 16.08 & 0 \\
NSGA-II & 0.60 & 9.20 & 4.99 & 25.34 & 1.75 & 0.70 & 0 \\
SPEA2 & 0.70 & 6.60 & 3.33 & 21.60 & 2.03 & 42.42 & 0 \\
AGA & 4.50 & 20.5 & 5.76 & 12.18 & 5.09 & 10.20 & 0 \\
Ideal & 26.0 & 22.9 & 1.84 & 05.82 & 243.60 & 95.05 & 0 \\
Dominating & 4.50 & 26.1 & 5.76 & 11.69 & 5.09 & 95.05 & 0 \\
ARA & 8.30 & 8.00 & 7.37 & 08.02 & 496.23 & 49.98 & 0.12 \\
Distributed & 22.6 & 24.6 & 4.28 & 12.82 & 122.76 & 47.52 & 0 \\
Distance & 56.7 & 65.5 & 9.69 & 12.82 & 411.99 & 47.52 & 0
\end{tabular}

Table 5. Time spent (seconds) by each archiving technique.

\begin{tabular}{lccccccc}
\hline Archiver & 1 & 2 & 3 & 4 & 5 & 6 & 7 \\
\hline Dominating & 0.03 & 0.04 & 0.00 & 0.03 & 0.00 & 0.01 & 0.01 \\
NSGA-II & 13.00 & 15.09 & 3.11 & 17.60 & 0.90 & 2.8 & 1.28 \\
SPEA2 & 12.93 & 14.88 & 2.71 & 14.65 & 1.11 & 2.54 & 1.40 \\
AGA & 0.11 & 0.14 & 0.03 & 0.15 & 0.02 & 0.08 & 0.01 \\
AA & 21.97 & 32.51 & 6.13 & 77.60 & 2.58 & 5.60 & 1.53 \\
MGA & 3.39 & 3.74 & 0.58 & 1.78 & 0.58 & 1.66 & 0.01 \\
Ideal & 0.66 & 0.76 & 0.12 & 0.74 & 0.08 & 0.21 & 0.04 \\
Distance & 0.83 & 1.06 & 0.18 & 1.05 & 0.12 & 0.36 & 0.04 \\
Distributed & 12.91 & 11.79 & 1.95 & 7.33 & 2.57 & 8.08 & 0.05 \\
ARA & 1.30 & 30.51 & 3.75 & 141.33 & 0.02 & 0.12 & 0.07 \\
\hline
\end{tabular}

\section{Recycling Solutions}

Based on the analysis shown in Section 3, this work proposes a deeper investigation on how helpful discarded solutions can be within an optimization process. The method consists of two steps. First, a recycle bin is created to store solutions rejected by the archiving technique used by an optimization algorithm. Including solutions in the recycle bin requires $O(1)$ processing time. No verification of Pareto dominance is done in this step. Periodically, the optimizer verifies the content of this bin and recovers good solutions. The goal is to improve the quality of the approximation set (population or external archive) along the optimization process. In this study, the population or external archive is notated as $P$ and the recycle bin as Bin. Since the archiving techniques may deteriorate or not $\subseteq Y^{*}$, there can be solutions in $P$ dominated by solutions in Bin. Therefore, this study proposes bringing solutions from Bin to $P,|P|=N$.

Periodically, the optimizer selects $N$ solutions from $\{P \cup$ $n d s(\operatorname{Bin})\}$ to maintain in $P$, where $n d s($ Bin $)$ stands for nondominated solutions from the recycling bin. There may be many ways of selecting these solutions. Three recycling methods are proposed and investigated in this study: (i) random selection, (ii) crowding distance selection and (iii) deterioration verification. Thereby, four scenarios are investigated for each optimizer: no recycling and one for each recycling method. The remainder of this section presents the recycling methods, the optimizers where they were implemented and the problems used in the experiments. 


\subsection{Recycling Methods}

Random selection, crowding distance and deterioration verification recycling methods are notated as random, crowdd and verif, respectively. Clean denotes the version of the optimizer without recycling. Algorithm 3 illustrates the use of a recycling method within an optimizer. The optimization process is executed in the process() procedure, for instance, the steps of a Genetic Algorithm. Solutions discarded by the archiving technique are sent to the recycling bin in Line 3. Recycling takes place in Lines 4-6. Every $K$ iterations dominated solutions in Bin are discarded and $P_{i+1}$ is updated by a recycling method. The proposed methods are described in the following where Bin stands for the recycling bin after dominated solutions are removed.

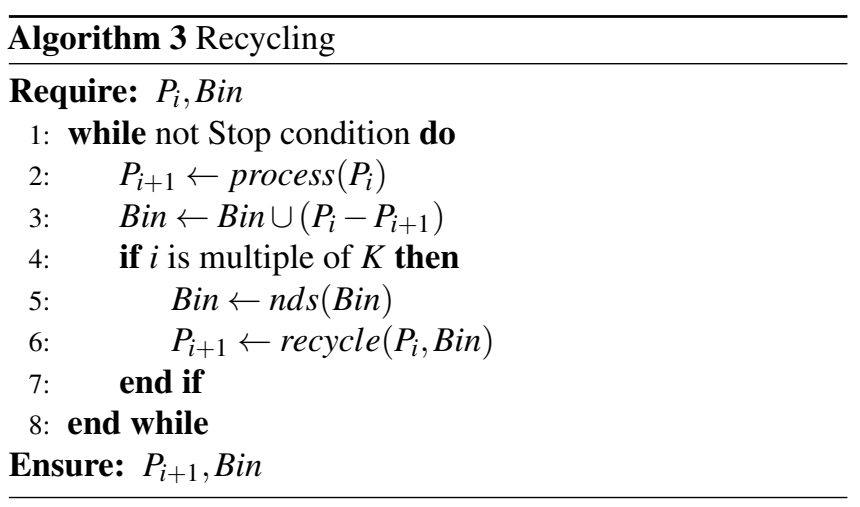

Random Selection: This method selects $N$ random points from $\left\{P_{i} \cup \operatorname{Bin}\right\}$.

Crowding Distance Selection: The best $N$ solutions according to crowding distance are selected. This metric was proposed by [10] in the NSGA-II algorithm to ensure diversity of the population. It assigns low priorities to solutions located in crowded regions of the objective space (see Algorithm 2).

Deterioration Verification: Given $P$ and Bin, the method verifies if each solution $P_{i} \in P$ is dominated by some solution in Bin, i.e., $\exists P_{i} \in P, \exists \operatorname{Bin}_{j} \in \operatorname{Bin}$ such that $\operatorname{Bin}_{j} \prec P_{i}$. If so, the dominated solution in $P$ is replaced by $B_{i n}$. In case of more than one solution in Bin dominate $P_{i}$, then $P_{i}$ is replaced by one of them selected randomly.

\section{Computational Experiments}

We investigated whether the recycling methods can improve the performance of some evolutionary optimizers concerning their outcomes. We did not focus on comparing distinct optimizers to specific problems. To evaluate the potential of the proposed recycling techniques, three well-known optimizers, NSGA-II [10], SPEA2 [1] and PAES [2], were tested on the benchmark problems presented in section 6.1. The SPEA2, NSGA-II and PAES implementations were obtained from the jMetal framework [18]. The experiments were executed on a PC with an Intel Core i7-3632QM processor and $2.2 \mathrm{GHz}$ of RAM, which ran Ubuntu 14.04 LTS 64Bits.

The stop criterion of the NSGA-II and the SPEA2 was 350 iterations. Once the PAES algorithm generates only one solution per iteration, its stop criterion was $350 *|N|$, where $|N|$ is the size of the population of the NSGA-II and the SPEA2, $|N|=50$. Default jMetal's mutation and crossover operations were used by the NSGA-II and the SPEA2. Recycling was executed every 50 iterations.

Each experiment (optimizer + archiving technique $\rightarrow$ problem) was executed 30 times, independently, with integer seeds for the random number generator from the range of $[1,30]$.

Hypervolume and $\varepsilon$-additive quality indicators were used to assess the approximation sets generated by each optimizer. The quality indicators were computed every 50 iterations of each execution. However, for the sake of space, we report the results computed for the final approximation sets, i.e., those obtained at the end of each run. If necessary, complete results are reported to clarify some behavior of a specific optimizer/problem. The Kruskal-Wallis (KW) test [19], at a significance level of 0.05 , was employed to detect differences in the behaviors of the optimizers. The reference set needed to compute the $\varepsilon$-add indicator consisted of the nondominated solutions from the 30 final approximation sets produced by each optimizer + archiving technique $\rightarrow$ problem. The reference point needed to compute the hypervolume indicator consisted of the worst values found for each objective. Thus, the reference point was dominated by all points of the reference set.

Section 6.1 presents the problems used in the experiments. Sections 6.2, 6.3 and 6.4 present the results of the experiments for the NSGA-II, SPEA2 and PAES, respectively.

\subsection{Problems}

Discrete and continuous benchmark problems were used in the experiments. The problems are described as follows.

\subsubsection{Knapsack Problem}

Given a set with $k$ items, $L=\left\{l_{1}, l_{2}, \ldots, l_{k}\right\}$, each item with a profit $p_{i}$ and a weight $w_{i}, i=1, \ldots, k$, and a knapsack with capacity $W$, the Knapsack Problem (KP) consists in selecting the items which maximize the total collected profit, so that the maximum weight $W$ is not exceeded. In the multiobjective version of the $\mathrm{KP}(\mathrm{mKP}), d$ profits and $d$ weights are associated with each item. There are $d$ knapsack capacities, $W_{1}, \ldots, W_{d}$. The mKP formulation is given in (2)-(4).

$$
\begin{aligned}
& \max f(x)=\sum_{i=1}^{k} x_{i} p_{1}^{i}, \sum_{i=1}^{k} x_{i} p_{2}^{i}, \ldots, \sum_{i=1}^{k} x^{i} p_{d}^{i} \\
& \text { subject to } \\
& \sum_{i=1}^{k} x_{i} w_{j}^{i} \leq W_{j} \quad \forall j \in \mathbb{N}, j=1, \ldots, d \\
& x_{i} \in\{0,1\}
\end{aligned}
$$


The mKP instances were generated with the random generator from PISA [20] which was implemented on the jMetal framework. Instances were generated with 100, 200, 500 and 750 items, 2, 3 and 4 objectives. The seed computed for the random generator was given by seed $=10 * k+d$, where $k$ is the number of items and $d$ the number of objectives. The mKP instances are named KS $k-d$.

\subsubsection{Quadratic Assignment Problem}

The quadratic assignment problem (QAP), as proposed by [21], consists in assigning $n$ industrial plants to $n$ locations, such that the cost of the flow of commodities supplied by one facility to another is minimized. The $m$ objective functions of a multi-objective QAP (mQAP), as presented by [22], are formulated in (5), where the $k$-th objective function is given in $(6), k=1, \ldots, m$.

$$
\begin{array}{r}
\min C(\phi)=\left\{C^{1}(\phi), C^{2}(\phi), \ldots, C^{m}(\phi)\right\} \\
C^{k}(\phi)=\sum_{i=1}^{n} \sum_{j=1}^{n} a_{i j} b_{\phi_{i}}^{k} \phi_{j}
\end{array}
$$

The mQAP instances were generated with the generator proposed by [22]. Instances were generated with 10, 20, 30 and 40 facilities and 2, 3 and 4 objectives. The mQAP instances are named mqapnndz, where $n n$ is the number of facilities, $d$ the number of objectives and $z$ the instance identification.

\subsubsection{Continuous Problems}

The benchmark continuous problems used in the experiments were: WFG [23], LZ09 [24], DTLZ [25], ZDT [26], Viennet2 and Viennet3, by [27], Fonseca [28] and Kursawe [29]. WFG problems were proposed to create a toolkit to generate multi-objective optimization problems with multiple and controlled features. On this toolkit, it is possible to add intended complexities to the problem, such as non-separability or multi-modality. Furthermore, it is possible to control the nature of the Pareto front, making it as linear, convex, concave, convex/concave and disconnected. The work of [23] provided 9 problems based on this toolkit, called WFG1-9. All those problems were tested in the experiments reported in this study. LZ09 problems were proposed by [24] to test variants of some multi-objective evolutionary algorithms.

\subsection{Recycling with the NSGA-II}

The NSGA-II (Non-dominating Sorting Genetic Algorithm II) is a multi-objective genetic algorithm proposed by [10]. It is one of most studied multi-objective algorithms in the literature. Initially a population, $P_{1}$, with $N$ individuals, is created and evaluated. Then, every new iteration genetic operators are applied to the individuals of the current population generating a new population, $Q$. The population of the $i$-th iteration, $P_{i+1}$, is obtained from $P_{i} \cup Q$. First, $P_{i} \cup Q$ is divided into $r$ subsets, $F_{k}, k=1, \ldots, r$, called fronts, such that $F_{1}$ contains the nondominated solutions of $P_{i} \cup Q$ and every element of
$F_{k}, k=2, \ldots, r$, is dominated by at least one element of $F_{k-1}$. Fronts $F_{k}$ are added to $P_{i+1}$ in ascending order of their indexes up to reaching $N$ individuals. In case $P_{i+1}$ is not completed after the insertion of the $k$ - 1 -th front and the $k$-th front does not fit completely in $P_{i+1}$, the solutions from $F_{k}$ with the largest values of crowding distance (Algorithm 2) complete the new population.

\subsection{1 mKP}

Table 6 shows the results of the NSGA-II and its variants with recycling techniques for $\mathrm{mKP}$ instances. The table shows the average hypervolume and $\varepsilon$-add, computed from the output of 30 independent executions of each NSGA-II variant. The columns show the instance name, the factor by which the hypervolume values have to be multiplied, and the results for each variant. Bold values indicate the best values for each quality indicator.

When compared with the clean version, the verif variant presented the best hypervolume results for 8 from the 12 instances tested, and the best $\varepsilon$-add values for 7 instances. The clean version was better than the crowdd and the random version for 8 instances concerning the hypervolume. According to the $\varepsilon$-add indicator the clean method achieved better results than the crowdd and random methods for 8 and 10 instances, respectively.

The statistical test showed that the variant with the random method performed worse than the other variants on instances KS100-3 and KS100-4. Significant differences between the variants were not indicated by the $\mathrm{KW}$ test for the remaining instances.

In average the clean, crowdd, random and verif variants spent $0.90,4.05,4.05$ and 4.04 seconds, respectively.

\subsection{2 mQAP}

Table 7 shows the average results of hypervolume and $\varepsilon$-add achieved by the NSGA-II variants for the mQAP.

The verif version presented the best results for 25 from the 36 instances tested regarding both quality indicators. The best performance of verif can be observed for the 3 and 4objective instances. The best results for the 12 2-objective instances, according to the hypervolume indicator, came from the random and clean methods. Differences due to the $\varepsilon$ add indicator were small for the 2-objective instances. The statistical test did not point out significant differences between the methods for the 2-objective instances.

Verif presented the best hypervolume and $\varepsilon$-add results for all 3 and 4-objective instances. Statistical differences between clean and verif were pointed out by the Kruskal-Wallis test for 21 instances. Statistical differences were also pointed out for verif and crowdd for all 3 and 4-objective instances. For 3 objective instances, the behaviors of verif and random were statistically different for 9 instances. Statistical differences between verif and random were not verified for 4-objective instances. A notable point concerns the instance size: the bigger the instance, the better is the performance of the verif and random methods. 
Investigation of Archiving Techniques for Evolutionary Multi-objective Optimizers

Table 6. Results of the NSGA-II variants for the mKP instances

\begin{tabular}{l|llccc|cccc}
\hline Inst & \multicolumn{5}{|c|}{ Hypervolume } & \multicolumn{4}{c}{$\varepsilon-a d d$} \\
\hline & factor & clean & crowdd & random & verif & clean & crowdd & random & verif \\
KS100-2 & $10^{5}$ & 1.461 & $\mathbf{1 . 4 9 5}$ & 1.426 & 1.464 & 123.6 & $\mathbf{1 1 2 . 5}$ & 128.2 & 121.0 \\
KS100-3 & $10^{8}$ & 2.386 & 2.298 & 2.189 & $\mathbf{2 . 4 8 2}$ & 216.0 & 221.0 & 266.6 & $\mathbf{2 1 3 . 7}$ \\
KS100-4 & $10^{11}$ & 1.931 & 1.535 & 1.865 & $\mathbf{2 . 0 6 6}$ & 265.8 & 307.2 & 290.1 & $\mathbf{2 4 9 . 6}$ \\
KS250-2 & $10^{8}$ & $\mathbf{2 . 5 4 5}$ & 2.344 & 2.209 & 2.224 & $\mathbf{4 0 6 . 5}$ & 408.4 & 430.4 & 450.7 \\
KS250-3 & $10^{8}$ & 4.687 & 4.587 & 4.884 & $\mathbf{5 . 0 6 4}$ & 457.8 & 433.6 & $\mathbf{4 2 5 . 1}$ & 437.2 \\
KS250-4 & $10^{11}$ & 7.074 & 6.005 & 6.159 & $\mathbf{7 . 2 8 4}$ & $\mathbf{4 9 7 . 7}$ & 539.4 & 552.6 & 515.5 \\
KS500-2 & $10^{5}$ & 1.635 & 2.033 & $\mathbf{2 . 2 4 8}$ & 2.238 & 647.4 & 571.8 & 576.4 & $\mathbf{5 4 4 . 3}$ \\
KS500-3 & $10^{8}$ & 1.662 & $\mathbf{1 . 8 8 8}$ & 1.174 & 1.461 & 878.8 & $\mathbf{8 2 1 . 5}$ & 900.7 & 841.1 \\
KS500-4 & $10^{11}$ & 7.351 & 7.608 & 8.126 & $\mathbf{8 . 4 1 4}$ & $\mathbf{8 0 7 . 5}$ & 809.6 & 822.3 & 820.0 \\
KS750-2 & $10^{4}$ & $\mathbf{9 . 3 9 1}$ & 6.316 & 6.089 & 4.086 & $\mathbf{9 2 6 . 7}$ & 1011.5 & 1050.1 & 1001.7 \\
KS750-3 & $10^{7}$ & $\mathbf{6 . 4 6 6}$ & 4.519 & 5.804 & 4.587 & $\mathbf{1 0 0 2 . 4}$ & 1099.4 & 1063.9 & 1030.0 \\
KS750-4 & $10^{12}$ & 1.471 & 0.964 & 1.571 & $\mathbf{2 . 0 5 4}$ & 1070.7 & 1180.3 & 1088.0 & $\mathbf{9 8 0 . 6}$ \\
\hline
\end{tabular}

Table 7. Results of the NSGA-II variants for the mQAP instances

\begin{tabular}{|c|c|c|c|c|c|c|c|c|c|}
\hline Inst & \multicolumn{5}{|c|}{ Hypervolume } & \multicolumn{4}{|c|}{$\varepsilon-a d d$} \\
\hline & factor & clean & crowdd & random & verif & clean & crowdd & random & verif \\
\hline mqap1021 & $10^{9}$ & 1.265 & 1.243 & 1.226 & 1.234 & 6204.5 & 6071.7 & 6814.7 & 6123.3 \\
\hline mqap1022 & $10^{9}$ & 2.501 & 2.422 & 2.431 & 2.460 & 4597.6 & 5672.5 & 5520.5 & 5309.3 \\
\hline mqap1023 & $10^{9}$ & 1.678 & 1.682 & 1.682 & 1.668 & 6286.3 & 6265.5 & 5913.8 & 6116.1 \\
\hline mqap2021 & $10^{10}$ & 1.154 & 1.142 & 1.168 & 1.172 & 33876.7 & 33572.3 & 33267.5 & 32931.9 \\
\hline mqap2022 & $10^{9}$ & 4.806 & 4.594 & 4.540 & 4.650 & 28539.5 & 29170.9 & 30489.5 & 31014.5 \\
\hline mqap2023 & $10^{10}$ & 1.195 & 1.129 & 1.203 & 1.189 & 30830.0 & 32658.5 & 28803.3 & 29777.5 \\
\hline mqap3021 & $10^{10}$ & 2.171 & 2.141 & 2.122 & 2.126 & 50318.6 & 54437.5 & 53842.9 & 51610.5 \\
\hline mqap3022 & $10^{10}$ & 3.272 & 3.274 & 3.328 & 3.292 & 75914.6 & 70599.4 & 72956.4 & 72440.7 \\
\hline mqap3023 & $10^{10}$ & 3.251 & 3.247 & 3.236 & 3.200 & 55244.6 & 50616.3 & 52529.9 & 55413.1 \\
\hline mqap4021 & $10^{10}$ & 6.167 & 6.092 & 6.449 & 6.394 & 87069.6 & 88120.1 & 85054.9 & 85965.6 \\
\hline mqap4022 & $10^{10}$ & 2.589 & 2.433 & 2.669 & 2.626 & 65178.8 & 69125.1 & 69172.5 & 69012.5 \\
\hline mqap4023 & $10^{10}$ & 2.363 & 2.115 & 2.464 & 2.361 & 74865.3 & 74701.3 & 75106.5 & 68633.5 \\
\hline mqap1031 & $10^{14}$ & 1.112 & 1.017 & 1.131 & 1.207 & 11496.4 & 15102.0 & 11046.8 & 9048.5 \\
\hline mqap1032 & $10^{14}$ & 1.255 & 1.080 & 1.310 & 1.399 & 11949.6 & 17103.2 & 11065.3 & 9142.2 \\
\hline mqap1033 & $10^{13}$ & 7.118 & 6.153 & 7.210 & 7.741 & 9970.8 & 13920.1 & 10381.0 & 8722.8 \\
\hline mqap2031 & $10^{15}$ & 2.757 & 2.505 & 2.951 & 3.016 & 49317.8 & 59213.5 & 50501.9 & 47373.2 \\
\hline mqap2032 & $10^{15}$ & 2.441 & 2.410 & 2.496 & 2.716 & 48570.4 & 53621.7 & 48060.7 & 42354.7 \\
\hline mqap2033 & $10^{15}$ & 1.928 & 1.658 & 1.999 & 2.261 & 47941.0 & 58536.1 & 49637.3 & 41672.8 \\
\hline mqap3031 & $10^{16}$ & 1.214 & 1.076 & 1.291 & 1.333 & 86366.4 & 104042.0 & 81343.5 & 77931.8 \\
\hline mqap3032 & $10^{16}$ & 1.086 & 0.981 & 1.108 & 1.277 & 86214.9 & 100114.0 & 81861.5 & 73268.8 \\
\hline mqap3033 & $10^{16}$ & 1.458 & 1.296 & 1.519 & 1.622 & 91467.9 & 105700.0 & 85098.5 & 80868.8 \\
\hline mqap4031 & $10^{16}$ & 3.012 & 2.650 & 3.362 & 3.559 & 130372.0 & 148274.0 & 117812.0 & 110192.0 \\
\hline mqap4032 & $10^{16}$ & 2.382 & 2.160 & 2.703 & 2.927 & 133825.0 & 146455.0 & 124835.0 & 117630.0 \\
\hline mqap4033 & $10^{16}$ & 4.324 & 4.190 & 4.821 & 5.123 & 146348.0 & 154876.0 & 130113.0 & 119700.0 \\
\hline mqap1041 & $10^{18}$ & 4.627 & 3.274 & 5.125 & 5.795 & 17486.8 & 23927.9 & 15413.7 & 13362.6 \\
\hline mqap1042 & $10^{18}$ & 6.470 & 4.447 & 7.964 & 8.803 & 19977.9 & 27342.9 & 16622.7 & 14682.1 \\
\hline mqap1043 & $10^{18}$ & 5.718 & 3.826 & 7.174 & 7.948 & 19052.5 & 26815.8 & 15452.4 & 13682.0 \\
\hline mqap2041 & $10^{20}$ & 4.346 & 3.869 & 5.272 & 5.715 & 72735.9 & 84229.3 & 62832.5 & 58426.7 \\
\hline mqap2042 & $10^{20}$ & 3.722 & 3.171 & 4.907 & 5.454 & 73489.3 & 82557.4 & 61448.9 & 57157.9 \\
\hline mqap2043 & $10^{20}$ & 6.145 & 5.482 & 7.318 & 8.313 & 68401.9 & 78180.9 & 61099.3 & 57071.7 \\
\hline mqap3041 & $10^{21}$ & 3.732 & 3.371 & 4.272 & 5.204 & 124921.0 & 133750.0 & 111912.0 & 100329.0 \\
\hline mqap3042 & $10^{21}$ & 2.689 & 2.480 & 3.557 & 3.958 & 128565.0 & 142696.0 & 112369.0 & 108146.0 \\
\hline mqap3043 & $10^{21}$ & 4.151 & 3.618 & 4.460 & 5.106 & 120551.0 & 134303.0 & 113570.0 & 105361.0 \\
\hline mqap4041 & $10^{22}$ & 1.982 & 1.755 & 2.268 & 2.553 & 188274.0 & 202735.0 & 169359.0 & 161579.0 \\
\hline mqap4042 & $10^{22}$ & 1.656 & 1.550 & 2.012 & 2.161 & 184825.0 & 196702.0 & 159827.0 & 157626.0 \\
\hline mqap4043 & $10^{22}$ & 1.974 & 1.844 & 2.404 & 2.584 & 190013.0 & 204006.0 & 160776.0 & 163512.0 \\
\hline
\end{tabular}

Figure 1 shows the hypervolumes calculated for 2 instances: mQAP4021, which has 40 facilities and 2 objectives, and mQAP4041, which has 40 facilities and 4 objectives. This figure illustrates the general behavior of the algorithm for 2 and 4-dimensional instances. For the 2-dimensional instance, the figure shows that the hypervolume curves of the
4 algorithmic variants are close one another. However, for 4-dimensional instances, the random and verif methods stood up along the optimization.

In average the clean, crowdd, random and verif variants spent $0.61,1.92,2.06$ and 2.08 seconds, respectively. When the number of objectives was 2 or 3 , crowdd method was faster 

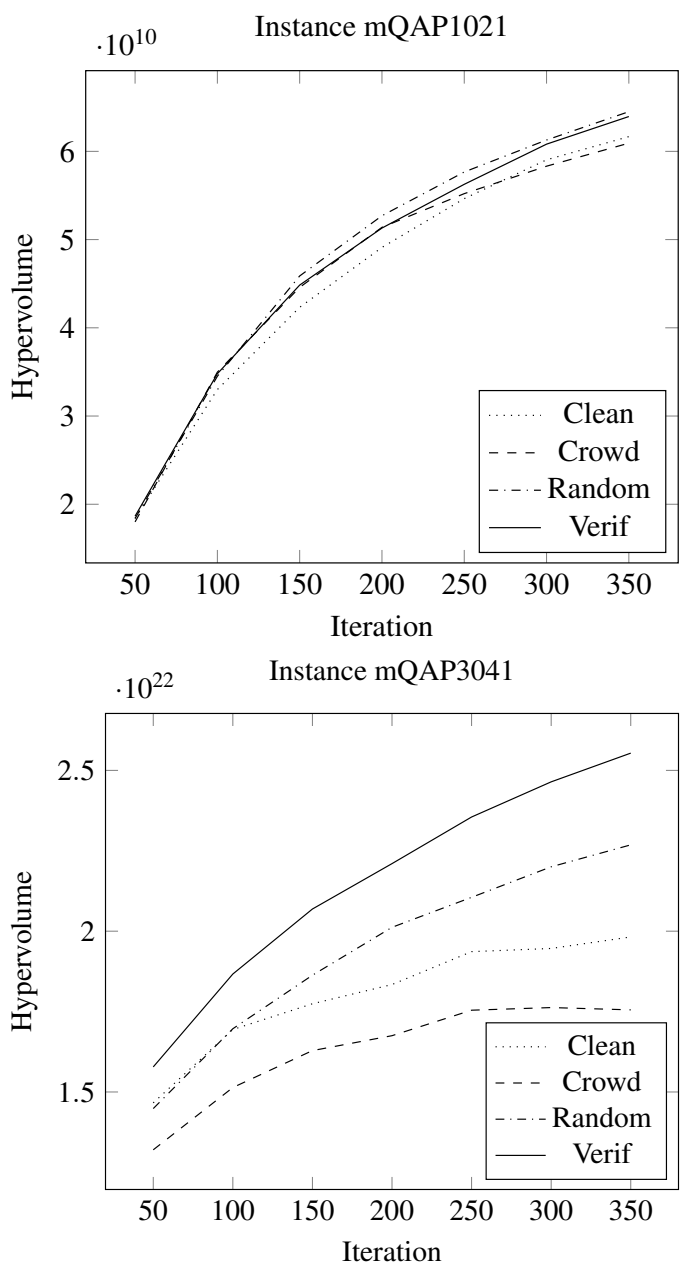

Figure 1. Hypervolume evolution calculated for the NSGA-II variants applied to the mQAP.

than random and verif, and spent from 0.2 to 0.4 seconds less.

\subsubsection{Continuous problems}

Table 8 shows the results of the NSGA-II variants for continuous instances. The DTLZ problems are named $\mathrm{DTlZN}{ }^{d}$, where $d$ is the number of objectives. The hypervolume values presented for the DTLZ instance has to be multiplied by $10^{11}$.

In general, the verif variant generated the best approximation sets, concerning the two quality indicators. The statistical tests for the hypervolume values indicated that the verif method outperformed the others on all 4-objective DTLZ instances and the DTLZ2 ${ }^{3}$, DTLZ5 ${ }^{2}$, LFZ09F1, LFZ09F6, WFG8, WFG9, Fonseca, Kursawe and Viennet3 instances. The crowdd and random methods were outperformed by the verif and clean on the DTLZ, Fonseca, Kursawe and Viennet3 instances. The statistical tests for the $\varepsilon$-add values indicated that the verif method outperformed the other methods on the DTLZ6 $^{4}$ and LFZ09F1 problems and that, in general, the crowdd and random methods were outperformed by the verif and clean.

In summary, for the continuous problems, the best results were obtained by the verif variant, followed by the standard
NSGA-II algorithm. The clean variant did not outperform the verif variant on any problem regarding both quality indicators. The crowdd method presented the worst results, in general.

The average times spent were $0.58,2.50,2.54$ and 2.60 seconds respectively by clean, crowdd, random and verif methods.

\subsubsection{Discussion about the NSGA-II variants results}

The crowdd method, based on the crowding distance brought no benefit to the NSGA-II. This fact showed that double applying the crowding distance to select nondominated solutions for the NSGA-II also magnified the deterioration effects. In general, the verif method improved the NSGA-II results regarding the discrete and continuous optimization problems concerning both quality indicators. The random method improved the NSGA-II results for the mQAP instances.

\subsection{Recycling with the SPEA2}

The SPEA2 (Strength Pareto Evolutionary Algorithm), proposed by [1], is also a multi-objective genetic algorithm. SPEA2 maintains a regular population and an external archive which stores the non-dominated solutions generated during the algorithm execution. The fitness of the solutions within the population and the external archive are computed every iteration. The fitness $F(i)$ of the $i$-th solution is the sum of two parcels: the ratio between the number of solutions dominated by the $i$-th solution and the population size, and an estimation based on the closeness among solutions. The aim of the latter parcel is to ensure diversity. The selection method of the SPEA2 is explained in Section 3.2.

\subsection{1 $\mathrm{mKP}$}

The results of the SPEA2 variants applied to the mKP are presented in Table 9.

Table 9 shows that the 3 recycling variants present better results for both indicators than the standard SPEA2 algorithm. The crowdd variant presented the best results for 6 and 8 instances from the 12 tested, regarding the hypervolume and $\varepsilon$-add indicators, respectively. The statistical test pointed out significant differences for the 3 recycling variants in comparison with the clean variant for the KS100-2 and KS100-4 instances. The crowdd variant was also statistically different from the clean variant on the KS100-3, KS250-2 and KS250 instances.

The average processing times were 2.01, 4.83, 4.84 and 4.94 seconds, for clean, crowdd, random and verif methods.

The quality indicators computed for the approximation sets generated by the SPEA2 applied to the mQAP are shown in Table 10.

\subsection{2 mQAP}

For the hypervolume indicator, Table 10 shows that except the mqap2021 instance, the best results for all instances were found by the recycling techniques. The KW test indicated favorable results over the clean version by some recycling technique on 23 instances. The crowdd version presented significant better results than the clean for 5 instances concerning 
Investigation of Archiving Techniques for Evolutionary Multi-objective Optimizers

Table 8. Results of the NSGA-II variants for the continuous instances

\begin{tabular}{|c|c|c|c|c|c|c|c|c|}
\hline Problem & \multicolumn{4}{|c|}{ Hypervolume } & \multicolumn{4}{|c|}{$\varepsilon-a d d$} \\
\hline & clean & crowdd & random & verif & clean & crowdd & random & verif \\
\hline DTLZ1 $^{2}$ & 0.115 & 0.110 & 0.102 & 0.119 & 0.0230 & 0.0515 & 0.0641 & 0.0173 \\
\hline DTLZ1 $^{3}$ & 0.046 & 0.057 & 0.056 & 0.064 & 0.2763 & 0.2036 & 0.2290 & 0.2110 \\
\hline DTLZ1 $^{4}$ & 118845 & 112984 & 120365 & 120641 & 5.7120 & 6.2058 & 5.8470 & 4.3925 \\
\hline DTLZ2 $^{2}$ & 0.204 & 0.183 & 0.201 & 0.205 & 0.0227 & 0.0766 & 0.0833 & 0.0211 \\
\hline DTLZ2 $^{3}$ & 0.499 & 0.471 & 0.494 & 0.511 & 0.1635 & 0.1883 & 0.2608 & 0.1671 \\
\hline DTLZ2 $^{4}$ & 4.899 & 4.398 & 4.743 & 4.998 & 0.2695 & 0.5183 & 0.3487 & 0.2586 \\
\hline DTLZ3 $^{2}$ & 0.021 & 0.007 & 0.005 & 0.003 & 2.3116 & 2.4806 & 2.1886 & 2.3804 \\
\hline DTLZ3 $^{3}$ & 220.954 & 168.196 & 189.918 & 184.941 & 7.0378 & 7.6683 & 8.1164 & 7.7836 \\
\hline DTLZ3 $^{4}$ & 1.845 & 1.82 & 1.847 & 1.847 & 73.0515 & 83.0216 & 62.0410 & 56.3366 \\
\hline DTLZ4 $^{2}$ & 0.095 & 0.086 & 0.094 & 0.095 & 0.5451 & 0.5673 & 0.5626 & 0.5439 \\
\hline DTLZ4 $^{3}$ & 0.324 & 0.307 & 0.326 & 0.332 & 0.3009 & 0.3224 & 0.3683 & 0.3039 \\
\hline DTLZ4 $^{4}$ & 0.875 & 0.690 & 0.864 & 0.915 & 0.3361 & 0.4354 & 0.4139 & 0.3225 \\
\hline DTLZ5 $^{2}$ & 0.204 & 0.180 & 0.201 & 0.205 & 0.0227 & 0.0859 & 0.0858 & 0.0209 \\
\hline DTLZ5 $^{3}$ & 0.044 & 0.038 & 0.043 & 0.045 & 0.0206 & 0.0920 & 0.0696 & 0.0204 \\
\hline DTLZ5 $^{4}$ & 6.876 & 6.679 & 6.024 & 6.936 & 0.1659 & 0.2709 & 0.2061 & 0.1442 \\
\hline DTLZ6 $^{2}$ & 0.393 & 0.371 & 0.391 & 0.386 & 0.0729 & 0.1202 & 0.0950 & 0.0757 \\
\hline DTLZ6 $^{3}$ & 4.042 & 2.588 & 3.065 & 4.413 & 0.5235 & 0.8267 & 0.6631 & 0.4796 \\
\hline DTLZ6 $^{4}$ & 6212.710 & 5687.100 & 5878.070 & 6699.650 & 2.3126 & 3.2298 & 2.7051 & 1.9240 \\
\hline DTLZ7 $^{2}$ & 0.458 & 0.450 & 0.449 & 0.459 & 0.1872 & 0.2044 & 0.2047 & 0.1857 \\
\hline DTLZ7 $^{3}$ & 0.772 & 0.723 & 0.754 & 0.806 & 0.6607 & 0.6949 & 0.7302 & 0.5958 \\
\hline DTLZ7 $^{4}$ & 4.583 & 4.136 & 4.478 & 4.852 & 0.6024 & 0.7392 & 0.7684 & 0.5531 \\
\hline LZ09F1 & 0.762 & 0.750 & 0.760 & 0.770 & 0.0352 & 0.0760 & 0.0515 & 0.0287 \\
\hline LZ09F2 & 0.475 & 0.478 & 0.474 & 0.478 & 0.2737 & 0.2701 & 0.2832 & 0.2707 \\
\hline LZ09F3 & 0.619 & 0.607 & 0.601 & 0.624 & 0.1882 & 0.1925 & 0.2323 & 0.1846 \\
\hline LZ09F4 & 0.770 & 0.740 & 0.754 & 0.773 & 0.1570 & 0.1749 & 0.1792 & 0.1565 \\
\hline LZ09F5 & 0.628 & 0.610 & 0.613 & 0.633 & 0.1373 & 0.1393 & 0.1658 & 0.1333 \\
\hline LZ09F6 & 1.507 & 1.411 & 1.516 & 1.518 & 0.0427 & 0.1292 & 0.0130 & 0.0059 \\
\hline LZ09F7 & 0.746 & 0.745 & 0.738 & 0.745 & 0.2795 & 0.2818 & 0.2882 & 0.2796 \\
\hline LZ09F8 & 1.815 & 1.813 & 1.818 & 1.815 & 0.3690 & 0.3735 & 0.3675 & 0.3690 \\
\hline LZ09F9 & 0.158 & 0.154 & 0.156 & 0.158 & 0.3130 & 0.3156 & 0.3227 & 0.3152 \\
\hline WFG1 & 34.723 & 34.836 & 30.488 & 35.026 & 0.5531 & 0.8562 & 0.7101 & 0.5650 \\
\hline WFG2 & 41.515 & 38.793 & 42.395 & 42.682 & 0.4678 & 0.8297 & 0.3874 & 0.4143 \\
\hline WFG3 & 15.896 & 12.485 & 14.763 & 16.185 & 0.3192 & 0.8679 & 0.3925 & 0.2875 \\
\hline WFG4 & 15.876 & 15.278 & 16.009 & 17.140 & 0.5579 & 0.6757 & 1.1390 & 0.4942 \\
\hline WFG5 & 16.366 & 15.643 & 16.468 & 16.964 & 0.5360 & 0.6357 & 0.8300 & 0.5177 \\
\hline WFG6 & 15.271 & 14.271 & 15.220 & 16.028 & 0.5590 & 0.6560 & 1.1504 & 0.5437 \\
\hline WFG7 & 15.435 & 15.325 & 16.390 & 17.058 & 0.5778 & 0.6297 & 0.9769 & 0.5185 \\
\hline WFG8 & 10.375 & 8.959 & 10.715 & 11.770 & 0.7698 & 0.8397 & 1.6226 & 0.6987 \\
\hline WFG9 & 15.459 & 14.517 & 15.916 & 16.477 & 0.5874 & 0.7013 & 0.9333 & 0.5253 \\
\hline Fonseca & 0.288 & 0.282 & 0.288 & 0.293 & 0.0261 & 0.0555 & 0.0481 & 0.0222 \\
\hline Kursawe & 193.376 & 192.945 & 191.612 & 193.533 & 0.1642 & 0.3216 & 0.9525 & 0.1422 \\
\hline Viennet 2 & 273.849 & 270.389 & 273.541 & 273.898 & 0.0537 & 0.2684 & 0.0309 & 0.0493 \\
\hline Viennet3 & 3.795 & 3.727 & 3.729 & 3.817 & 0.0737 & 0.1261 & 0.1381 & 0.0594 \\
\hline
\end{tabular}

Table 9. Results of the SPEA2 variants for the mKP instances

\begin{tabular}{l|ccccc|cccc}
\hline Inst & \multicolumn{5}{|c|}{ Hypervolume } & \multicolumn{4}{c}{$\varepsilon-$ add } \\
KS100-2 & factor & clean & crowdd & random & verif & clean & crowdd & random & verif \\
KS100-3 & $10^{5}$ & 0.955 & $\mathbf{1 . 1 9 8}$ & 1.102 & 1.119 & 200.0 & $\mathbf{1 2 8 . 8}$ & 155.2 & 148.3 \\
KS100-4 & $10^{10}$ & 5.612 & 7.778 & 7.995 & $\mathbf{8 . 2 3 7}$ & 297.9 & $\mathbf{2 2 6 . 3}$ & 242.9 & 233.7 \\
KS250-2 & $10^{5}$ & 4.278 & $\mathbf{5 . 0 6 1}$ & 4.794 & 4.729 & 432.1 & 354.8 & 381.8 & $\mathbf{3 4 7 . 3}$ \\
KS250-3 & $10^{8}$ & 1.392 & $\mathbf{1 . 6 0 8}$ & 1.504 & 1.338 & 410.8 & $\mathbf{3 6 5 . 8}$ & 385.2 & 403.2 \\
KS250-4 & $10^{11}$ & 1.306 & $\mathbf{1 . 7 9 1}$ & 1.603 & 1.679 & 554.1 & $\mathbf{4 4 1 . 6}$ & 486.6 & 460.7 \\
KS500-2 & $10^{5}$ & 1.807 & $\mathbf{1 . 9 4 6}$ & 1.579 & 1.646 & 678.7 & $\mathbf{6 7 2 . 3}$ & 708.9 & 725.0 \\
KS500-3 & $10^{8}$ & 1.602 & 1.556 & $\mathbf{1 . 6 2 0}$ & 1.583 & 818.1 & $\mathbf{7 1 7 . 3}$ & 803.8 & 762.4 \\
KS500-4 & $10^{12}$ & 1.293 & 1.498 & 1.457 & $\mathbf{1 . 5 4 2}$ & 867.8 & 841.1 & 881.8 & $\mathbf{8 0 2 . 2}$ \\
KS750-2 & $10^{4}$ & 2.865 & 2.605 & $\mathbf{6 . 0 9 6}$ & 3.073 & 1066.6 & 1026.6 & $\mathbf{9 7 8 . 6}$ & 1048.8 \\
KS750-3 & $10^{8}$ & 2.880 & 3.254 & 3.361 & $\mathbf{3 . 5 8 1}$ & 1172.5 & 1079.3 & 1098.5 & $\mathbf{1 0 2 2 . 3}$ \\
KS750-4 & $10^{11}$ & 4.992 & 5.582 & $\mathbf{5 . 6 7 5}$ & 5.398 & 1030.9 & $\mathbf{9 7 2 . 2}$ & 1017.1 & 1005.2 \\
\hline
\end{tabular}

the two indicators and for one more instance concerning the $\varepsilon$ add. The random version also outperformed the clean version on 12 instances concerning the two indicators and another instance according to the hypervolume. Finally, the verif versions presented better results than the clean version on 18 instances concerning both quality indicators and on other 3 
Investigation of Archiving Techniques for Evolutionary Multi-objective Optimizers

Table 10. Results of the SPEA2 variants for the mQAP instances

\begin{tabular}{|c|c|c|c|c|c|c|c|c|c|}
\hline Inst & & & Aypervolu & & & & & & \\
\hline & factor & clean & crowdd & random & verif & clean & crowdd & random & verif \\
\hline mqap1021 & $10^{9}$ & 1.055 & 1.203 & 1.187 & 1.191 & 12731.7 & 6778.6 & 7822.2 & 8757.2 \\
\hline mqap1022 & $10^{9}$ & 2.223 & 2.373 & 2.404 & 2.341 & 9477.8 & 6421.5 & 6427.8 & 8099.4 \\
\hline mqap1023 & $10^{9}$ & 1.465 & 1.611 & 1.606 & 1.571 & 10436.9 & 6647.5 & 7593.6 & 8633.2 \\
\hline mqap2021 & $10^{9}$ & 8.777 & 9.781 & 9.700 & 9.180 & 45727.5 & 35309.4 & 35960.7 & 40539.2 \\
\hline mqap2022 & $10^{10}$ & 1.111 & 1.184 & 1.195 & 1.166 & 38045.7 & 30397.5 & 31488.1 & 33253.5 \\
\hline mqap2023 & $10^{9}$ & 9.086 & 9.466 & 9.781 & 9.403 & 38005.5 & 32716.0 & 31860.1 & 35219.7 \\
\hline mqap3021 & $10^{10}$ & 2.027 & 1.965 & 1.981 & 1.962 & 74739.5 & 70833.0 & 72360.9 & 80784.7 \\
\hline mqap3022 & $10^{10}$ & 0.996 & 1.054 & 1.009 & 1.085 & 62192.5 & 57116.4 & 60214.8 & 60118.9 \\
\hline mqap3023 & $10^{10}$ & 2.220 & 2.101 & 2.290 & 2.270 & 58264.9 & 59330.5 & 52420.7 & 57003.6 \\
\hline mqap4021 & $10^{10}$ & 4.574 & 4.820 & 4.849 & 4.981 & 105791.0 & 87615.4 & 92224.7 & 93872.7 \\
\hline mqap4022 & $10^{10}$ & 3.225 & 3.467 & 3.427 & 3.350 & 90981.0 & 80097.7 & 88521.1 & 89989.6 \\
\hline mqap4023 & $10^{10}$ & 3.607 & 3.512 & 3.570 & 3.711 & 102051.0 & 104229.0 & 97381.0 & 99586.8 \\
\hline mqap1031 & $10^{14}$ & 1.058 & 1.119 & 1.183 & 1.191 & 12847.3 & 12191.2 & 9835.3 & 9636.1 \\
\hline mqap1032 & $10^{14}$ & 1.186 & 1.261 & 1.347 & 1.373 & 13340.4 & 13783.9 & 10429.7 & 10092.7 \\
\hline mqap1033 & $10^{13}$ & 6.633 & 6.877 & 7.265 & 7.455 & 12324.9 & 11393.5 & 10440.6 & 10015.3 \\
\hline mqap2031 & $10^{15}$ & 1.532 & 1.636 & 1.743 & 1.660 & 48522.8 & 44335.5 & 43491.7 & 46268.1 \\
\hline mqap2032 & $10^{15}$ & 1.107 & 1.292 & 1.284 & 1.370 & 48882.9 & 43313.7 & 43473.1 & 41757.0 \\
\hline mqap2033 & $10^{15}$ & 1.219 & 1.342 & 1.416 & 1.390 & 51228.5 & 41695.2 & 43473.8 & 43132.7 \\
\hline mqap3031 & $10^{16}$ & 1.017 & 1.111 & 1.084 & 1.120 & 74276.7 & 68915.0 & 67925.1 & 67626.6 \\
\hline mqap3032 & $10^{15}$ & 5.172 & 5.196 & 6.113 & 6.131 & 80058.1 & 76521.7 & 67754.0 & 65246.9 \\
\hline mqap3033 & $10^{15}$ & 5.387 & 6.167 & 6.149 & 6.292 & 83869.3 & 69493.7 & 75876.2 & 75592.3 \\
\hline mqap4031 & $10^{16}$ & 2.224 & 2.452 & 2.453 & 2.564 & 116142.0 & 104134.0 & 105021.0 & 107066.0 \\
\hline mqap4032 & $10^{16}$ & 1.118 & 1.219 & 1.237 & 1.203 & 112239.0 & 96531.4 & 100374.0 & 112237.0 \\
\hline mqap4033 & $10^{16}$ & 1.253 & 1.308 & 1.397 & 1.396 & 110470.0 & 100497.0 & 100212.0 & 104495.0 \\
\hline mqap1041 & $10^{18}$ & 4.286 & 4.598 & 5.638 & 5.920 & 18181.0 & 18667.2 & 14001.2 & 13424.7 \\
\hline mqap1042 & $10^{18}$ & 6.561 & 6.633 & 8.537 & 8.944 & 20229.7 & 21937.7 & 15211.5 & 15769.6 \\
\hline mqap1043 & $10^{18}$ & 5.053 & 4.932 & 6.834 & 7.182 & 19564.9 & 21424.3 & 14702.4 & 13881.1 \\
\hline mqap2041 & $10^{20}$ & 2.692 & 2.614 & 3.115 & 3.478 & 59898.3 & 64039.1 & 52576.8 & 50855.3 \\
\hline mqap2042 & $10^{20}$ & 2.646 & 2.819 & 3.152 & 3.385 & 65731.6 & 59361.9 & 54420.3 & 57334.7 \\
\hline mqap2043 & $10^{20}$ & 1.832 & 1.680 & 2.356 & 2.667 & 61901.1 & 64503.7 & 50726.0 & 50522.9 \\
\hline mqap3041 & $10^{21}$ & 1.471 & 1.222 & 1.683 & 1.786 & 98014.9 & 108609.0 & 85961.4 & 85391.7 \\
\hline mqap3042 & $10^{21}$ & 1.511 & 1.504 & 1.606 & 1.900 & 94906.7 & 100527.0 & 88206.8 & 83963.2 \\
\hline mqap3043 & $10^{21}$ & 0.766 & 0.612 & 0.798 & 1.040 & 103001.0 & 107707.0 & 91535.1 & 90588.9 \\
\hline mqap4041 & $10^{21}$ & 4.606 & 4.110 & 5.653 & 7.223 & 155401.0 & 168194.0 & 134847.0 & 129146.0 \\
\hline mqap4042 & $10^{21}$ & 4.256 & 4.083 & 4.451 & 5.855 & 136026.0 & 143045.0 & 126956.0 & 122154.0 \\
\hline mqap4043 & $10^{21}$ & 6.388 & 5.930 & 5.946 & 8.322 & 136890.0 & 148388.0 & 136348.0 & 120104.0 \\
\hline
\end{tabular}

instances concerning the hypervolume. The KW test did not point out best significant results for the clean version on any instance.

The major contributions for the standard algorithm came from the crowdd method on small instances and the verif method on large instances.

On mQAP instances, the clean method spent, in average, $2.2 \mathrm{~s}$ when $d=2$ and $d=4$, and $1.75 \mathrm{~s}$ when $d=3$. The average processing times of the recycling methods were 2.01, $4.04,3.91$ and 4.03, for clean, crowdd, random and verif, respectively.

\subsubsection{Continuous Problems}

Table 11 presents the results of the SPEA2 variants for the continuous problems. DTLZ1 $1^{4}$ hypervolumes have to be multiplied by $10^{6}$, DTLZ3 3 by $10^{5}$ and DTLZ3 ${ }^{4}$ by $10^{11}$.

Concerning the hypervolume indicator, Table 11 shows that a recycling technique produced the best results. Significant statistical differences between the verif and clean methods were pointed out by the KW test for 16 from 21 DTLZ instances, 3 from 9 LZ09 instances, 8 from 9 WFG instances and for the Fonseca, Kursawe and Viennet 3 instances. Signif- icant differences were found for 4 DTLZ instances, 1 LZ09 instance and 2 WFG instances between clean and random, and for 2 LF09 instances between crowdd and clean.

Concerning the $\varepsilon$-add indicator, the recycling techniques presented the best results for 17 DTLZ instances. Significant differences were pointed out in favor of the verif method compared with the clean method for 7 DTLZ instances, 1 LZ09 instance, 3 WFG instances and for the Fonseca, Kursawe, Viennet 2 and Viennet 3 instances. Significantly favorable results for the crowdd method compared with the clean method were observed for 3 LZ09 instances and 4 WFG instances. The random method was statistically better than the clean method for 1 LZ09 instance and 1 WFG instance.

The average processing times of the recycling methods were $1.70,4.00,3.99$ and 4.08 seconds for clean, crowdd, random and verif respectively.

\subsubsection{Discussion about the SPEA2 variants results}

The proposed recycling techniques significantly improved the SPEA2 results. Different from the NSGA-II, the crowdd method improved the outcome of the optimizer. We can note that crowdd method outperformed the other methods for half 
Investigation of Archiving Techniques for Evolutionary Multi-objective Optimizers

Table 11. Results of the SPEA2 variants for the continuous problems

\begin{tabular}{|c|c|c|c|c|c|c|c|c|}
\hline Problem & \multicolumn{4}{|c|}{ Hypervolume } & \multicolumn{4}{|c|}{$\varepsilon-a d d$} \\
\hline & clean & crowdd & random & verif & clean & crowdd & random & verif \\
\hline DTLZ1 $^{2}$ & 0.099 & 0.092 & 0.109 & 0.105 & 0.0861 & 0.1174 & 0.0528 & 0.0666 \\
\hline DTLZ $^{3}$ & 58.952 & 58.685 & 59.245 & 59.247 & 0.1627 & 0.5957 & 0.1395 & 0.1383 \\
\hline DTLZ1 $^{4}$ & 2.976 & 2.823 & 3.010 & 3.010 & 9.1186 & 19.9041 & 3.1314 & 2.4190 \\
\hline DTLZ2 $^{2}$ & 0.203 & 0.192 & 0.210 & 0.208 & 0.0618 & 0.0787 & 0.1046 & 0.0498 \\
\hline DTLZ2 $^{3}$ & 0.948 & 0.918 & 0.952 & 0.985 & 0.2162 & 0.2303 & 0.2452 & 0.2074 \\
\hline DTLZ2 $^{4}$ & 3.432 & 3.178 & 3.465 & 3.577 & 0.3661 & 0.4882 & 0.3773 & 0.3426 \\
\hline DTLZ3 $^{2}$ & 0.093 & 0.029 & 0.228 & 0.077 & 3.1664 & 3.9021 & 3.3059 & 3.3409 \\
\hline DTLZ3 $^{3}$ & 1.212 & 1.214 & 1.218 & 1.217 & 7.2194 & 6.4928 & 6.1686 & 5.9910 \\
\hline DTLZ3 $^{4}$ & 3.66164 & 3.61221 & 3.6666 & 3.6667 & 69.7490 & 138.0700 & 41.0422 & 35.9283 \\
\hline DTLZ4 $^{2}$ & 0.117 & 0.111 & 0.121 & 0.120 & 0.4287 & 0.4438 & 0.4601 & 0.4333 \\
\hline DTLZ4 $^{3}$ & 0.332 & 0.305 & 0.338 & 0.343 & 0.5943 & 0.6106 & 0.5923 & 0.5707 \\
\hline DTLZ4 $^{4}$ & 0.623 & 0.399 & 0.642 & 0.665 & 0.6043 & 0.7242 & 0.6054 & 0.6054 \\
\hline DTLZ5 $^{2}$ & 0.205 & 0.190 & 0.212 & 0.209 & 0.0618 & 0.0853 & 0.0869 & 0.0584 \\
\hline DTLZ5 $^{3}$ & 0.042 & 0.036 & 0.043 & 0.043 & 0.0492 & 0.1379 & 0.0750 & 0.0455 \\
\hline DTLZ5 $^{4}$ & 2.122 & 2.810 & 1.532 & 2.967 & 0.4450 & 0.3528 & 0.5567 & 0.2208 \\
\hline DTLZ6 $^{2}$ & 0.536 & 0.538 & 0.554 & 0.553 & 0.1575 & 0.1582 & 0.1374 & 0.1243 \\
\hline DTLZ6 $^{3}$ & 38.137 & 34.246 & 38.476 & 40.122 & 0.5470 & 0.9272 & 0.5263 & 0.3823 \\
\hline DTLZ6 $^{4}$ & 4130.730 & 3276.050 & 4189.770 & 4569.720 & 1.9922 & 3.8643 & 2.0774 & 1.4956 \\
\hline DTLZ7 $^{2}$ & 0.503 & 0.512 & 0.516 & 0.516 & 0.0678 & 0.0518 & 0.0488 & 0.0600 \\
\hline DTLZ7 $^{3}$ & 1.256 & 1.186 & 1.268 & 1.332 & 0.5455 & 0.5001 & 0.5352 & 0.4782 \\
\hline DTLZ7 $^{4}$ & 8.456 & 8.349 & 8.849 & 9.082 & 1.3501 & 1.0824 & 1.2966 & 1.1894 \\
\hline LZ09F1 & 0.632 & 0.640 & 0.670 & 0.674 & 0.0987 & 0.1165 & 0.0794 & 0.0634 \\
\hline LZ09F2 & 0.475 & 0.482 & 0.484 & 0.478 & 0.2719 & 0.2545 & 0.2569 & 0.2658 \\
\hline LZ09F3 & 0.659 & 0.684 & 0.669 & 0.686 & 0.2035 & 0.1572 & 0.2092 & 0.1829 \\
\hline LZ09F4 & 0.572 & 0.573 & 0.585 & 0.589 & 0.1049 & 0.1131 & 0.0977 & 0.0936 \\
\hline LZ09F5 & 0.675 & 0.694 & 0.681 & 0.696 & 0.1776 & 0.1313 & 0.1939 & 0.1598 \\
\hline LZ09F6 & 0.003 & 0.003 & 0.003 & 0.003 & 0.0018 & 0.0111 & 0.0000 & 0.0000 \\
\hline LZ09F7 & 1.551 & 1.624 & 1.606 & 1.618 & 0.4130 & 0.3758 & 0.3779 & 0.3768 \\
\hline LZ09F8 & 3.360 & 3.396 & 3.400 & 3.372 & 0.3665 & 0.3471 & 0.3505 & 0.3526 \\
\hline LZ09F9 & 0.159 & 0.156 & 0.157 & 0.162 & 0.3393 & 0.2865 & 0.3154 & 0.3043 \\
\hline WFG1 & 8.439 & 8.790 & 9.778 & 10.155 & 0.8048 & 0.8501 & 0.7673 & 0.6838 \\
\hline WFG2 & 39.378 & 33.672 & 41.186 & 41.334 & 0.5813 & 1.1026 & 0.3790 & 0.4481 \\
\hline WFG3 & 15.100 & 13.584 & 13.664 & 16.486 & 0.4802 & 0.8683 & 0.7779 & 0.3827 \\
\hline WFG4 & 14.412 & 15.258 & 15.920 & 16.341 & 0.8731 & 0.6735 & 0.9368 & 0.8216 \\
\hline WFG5 & 15.512 & 15.311 & 16.447 & 16.483 & 0.8934 & 0.6152 & 0.9068 & 0.7804 \\
\hline WFG6 & 14.924 & 15.115 & 15.971 & 16.509 & 0.9301 & 0.6055 & 1.1220 & 0.7971 \\
\hline WFG7 & 14.285 & 14.816 & 16.115 & 16.332 & 0.8297 & 0.6525 & 0.9764 & 0.7684 \\
\hline WFG8 & 11.384 & 10.737 & 13.651 & 14.180 & 1.1224 & 0.8909 & 1.3733 & 0.8754 \\
\hline WFG9 & 15.305 & 14.475 & 16.584 & 16.669 & 0.8075 & 0.6836 & 0.9939 & 0.7249 \\
\hline Fonseca & 0.265 & 0.281 & 0.288 & 0.289 & 0.0945 & 0.0545 & 0.0570 & 0.0648 \\
\hline Kursawe & 183.549 & 191.301 & 190.430 & 188.437 & 0.7915 & 0.6499 & 0.9770 & 0.6302 \\
\hline Viennet2 & 272.689 & 269.572 & 272.406 & 273.075 & 0.0462 & 0.2678 & 0.0307 & 0.0321 \\
\hline Viennet3 & 3.442 & 3.592 & 3.421 & 3.776 & 0.2944 & 0.2162 & 0.3065 & 0.1169 \\
\hline
\end{tabular}

of the mKP instances concerning both quality indicators. Verif was the best method for the other problems. It obtained the best indicators values on most problems. The random method also improved the SPEA2 results for several instances, and in general, had better results than the clean SPEA2.

\subsection{Recycling with PAES}

PAES (Pareto Archived Evolution Strategy) is a multi-objective evolution strategy proposed by [2]. This algorithm is based on local search and maintains an external archive that stores nondominated solutions generated during its execution. The AGA technique is used to filter new solutions which are candidates to be inserted into the external archive. PAES has the three following main components. A solution generator which mutates a guide solution employing local search and generates a new candidate solution. A solution selector that determines which solution, among the guide and the new one, is maintained for the next iteration. Finally, PAES maintains an external archive. As shown by [8], the external archive may deteriorate.

\subsection{1 mKP}

Table 12 shows the results of PAES $\rightarrow$ mKP. From the 12 $\mathrm{mKP}$ instances, the recycling techniques produced better hypervolume and $\varepsilon$-add values than the clean version for 10 instances. The statistical test pointed out a significant difference in favor of the random method compared with the clean version for instances KS100-4 and KS750-3.

The average times were $0.57,0.61,0.60$, and 0.61 for the clean, random, crowdd and verif methods, respectively. The average processing times of the recycling methods were slightly greater than the clean method. This fact might be happened due to the solutions generated by the mutation process were not as good as the guide one, and the recycling bin stored few solutions. 
Table 12. Results of the PAES variants for the mKP

\begin{tabular}{l|ccccc|cccc}
\hline Inst & \multicolumn{5}{|c|}{ Hypervolume } & \multicolumn{4}{c}{$\varepsilon-a d d$} \\
\hline & factor & clean & crowdd & random & verif & clean & crowdd & random & verif \\
KS100-2 & $10^{4}$ & 5.955 & 6.456 & $\mathbf{6 . 9 4 9}$ & 6.226 & 266.6 & $\mathbf{2 4 1 . 0}$ & 248.0 & 251.1 \\
KS100-3 & $10^{7}$ & 4.631 & 4.471 & $\mathbf{4 . 9 4 2}$ & 4.279 & 413.7 & 418.0 & $\mathbf{4 0 9 . 7}$ & 429.0 \\
KS100-4 & $10^{9}$ & 2.183 & 5.036 & $\mathbf{5 . 7 3 1}$ & 3.415 & 461.2 & 426.2 & $\mathbf{3 9 2 . 0}$ & 425.8 \\
KS250-2 & $10^{5}$ & $\mathbf{1 . 8 4 3}$ & 1.766 & 1.794 & 1.804 & $\mathbf{4 2 1 . 8}$ & 444.9 & 442.1 & 430.6 \\
KS250-3 & $10^{7}$ & 0.845 & 1.090 & 1.316 & $\mathbf{1 . 3 8 8}$ & 629.2 & 588.0 & $\mathbf{5 6 9 . 3}$ & 579.9 \\
KS250-4 & $10^{10}$ & $\mathbf{4 . 8 2 8}$ & 4.727 & 3.808 & 4.114 & 711.8 & $\mathbf{6 9 3 . 2}$ & 758.1 & 735.0 \\
KS500-2 & $10^{4}$ & 6.011 & 5.557 & $\mathbf{7 . 1 0 2}$ & 6.068 & 682.9 & 693.8 & $\mathbf{6 4 6 . 6}$ & 647.8 \\
KS500-3 & $10^{8}$ & 1.447 & 1.512 & $\mathbf{9 . 4 7 9}$ & 1.494 & $\mathbf{7 9 0 . 4}$ & 850.0 & 882.7 & 835.6 \\
KS500-4 & $10^{9}$ & 0.682 & $\mathbf{2 . 1 6 1}$ & 1.063 & 0.877 & 980.9 & 893.9 & 953.4 & $\mathbf{8 8 1 . 0}$ \\
KS750-2 & $10^{4}$ & 4.478 & $\mathbf{7 . 5 1 0}$ & 5.263 & 2.885 & 988.5 & 1014.0 & $\mathbf{9 7 3 . 7}$ & 1119.7 \\
KS750-3 & $10^{8}$ & 1.547 & 1.802 & $\mathbf{3 . 7 0 6}$ & 2.286 & 1461.9 & 1389.9 & $\mathbf{1 2 1 3 . 8}$ & 1349.2 \\
KS750-4 & $10^{11}$ & 6.118 & 5.489 & 5.445 & $\mathbf{6 . 7 8 1}$ & 1517.9 & 1533.6 & 1490.6 & $\mathbf{1 4 4 2 . 4}$ \\
\hline
\end{tabular}

Table 13. Results of the PAES variants for the mQAP

\begin{tabular}{|c|c|c|c|c|c|c|c|c|c|}
\hline Inst & \multicolumn{5}{|c|}{ Hypervolume } & \multicolumn{4}{|c|}{$\varepsilon-a d d$} \\
\hline & factor & clean & crowdd & random & verif & clean & crowdd & random & verif \\
\hline mqap1021 & $10^{8}$ & 3.712 & 3.997 & 4.168 & 4.351 & 19760.4 & 18919.0 & 18322.3 & 19337.4 \\
\hline mqap1022 & $10^{9}$ & 1.475 & 1.436 & 1.517 & 1.522 & 23532.5 & 23215.3 & 22143.9 & 22420.5 \\
\hline mqap1023 & $10^{8}$ & 6.717 & 6.875 & 7.015 & 6.668 & 16598.5 & 15882.9 & 15647.1 & 16757.4 \\
\hline mqap2021 & $10^{9}$ & 1.962 & 1.659 & 1.862 & 1.819 & 39391.2 & 42730.6 & 41038.1 & 41517.4 \\
\hline mqap2022 & $10^{8}$ & 6.284 & 5.353 & 5.587 & 2.838 & 36812.7 & 38162.1 & 36073.0 & 40879.1 \\
\hline mqap2023 & $10^{9}$ & 0.992 & 0.794 & 1.078 & 0.810 & 49910.3 & 48299.1 & 47905.8 & 47281.3 \\
\hline mqap3021 & $10^{9}$ & 7.088 & 7.827 & 8.892 & 7.260 & 74147.3 & 70077.1 & 66773.6 & 79268.7 \\
\hline mqap3022 & $10^{9}$ & 6.697 & 6.295 & 6.675 & 6.243 & 69962.4 & 71006.8 & 73399.5 & 71774.5 \\
\hline mqap3023 & $10^{9}$ & 1.470 & 2.448 & 2.185 & 3.369 & 68988.1 & 63734.2 & 67674.4 & 61112.6 \\
\hline mqap4021 & $10^{10}$ & 1.053 & 1.174 & 1.199 & 1.238 & 101063.0 & 104857.0 & 96199.0 & 101456.0 \\
\hline mqap4022 & $10^{10}$ & 1.472 & 1.419 & 1.410 & 1.475 & 102192.0 & 91813.0 & 100846.0 & 95384.4 \\
\hline mqap4023 & $10^{10}$ & 1.801 & 1.729 & 1.678 & 1.838 & 125341.0 & 122797.0 & 114639.0 & 115037.0 \\
\hline mqap1031 & $10^{13}$ & 2.727 & 2.703 & 2.809 & 2.588 & 20508.1 & 19926.5 & 18477.4 & 19914.4 \\
\hline mqap1032 & $10^{13}$ & 4.476 & 3.984 & 4.244 & 4.168 & 18874.2 & 21172.0 & 19410.7 & 20463.0 \\
\hline mqap1033 & $10^{13}$ & 1.433 & 1.569 & 1.472 & 1.463 & 20413.6 & 19743.6 & 21317.5 & 20035.0 \\
\hline mqap2031 & $10^{14}$ & 3.823 & 4.972 & 3.871 & 4.492 & 59236.7 & 54208.1 & 58907.5 & 56134.0 \\
\hline mqap2032 & $10^{14}$ & 5.754 & 5.744 & 5.216 & 5.084 & 61505.1 & 61398.8 & 64693.1 & 64012.6 \\
\hline mqap2033 & $10^{14}$ & 4.395 & 4.767 & 4.064 & 4.590 & 55399.2 & 53976.7 & 58613.3 & 53376.9 \\
\hline mqap3031 & $10^{15}$ & 1.694 & 1.862 & 1.889 & 1.838 & 93889.8 & 91113.3 & 91770.8 & 97319.1 \\
\hline mqap3032 & $10^{15}$ & 1.677 & 1.522 & 1.490 & 1.668 & 99701.2 & 99745.5 & 97415.5 & 97458.3 \\
\hline mqap3033 & $10^{14}$ & 4.895 & 4.570 & 3.938 & 5.400 & 96976.6 & 97384.4 & 101892.0 & 90340.0 \\
\hline mqap4031 & $10^{15}$ & 1.911 & 1.991 & 1.845 & 1.864 & 149312.0 & 140527.0 & 148034.0 & 143214.0 \\
\hline mqap4032 & $10^{15}$ & 3.808 & 4.117 & 3.940 & 4.036 & 131867.0 & 121285.0 & 131277.0 & 123708.0 \\
\hline mqap4033 & $10^{15}$ & 1.893 & 1.727 & 2.096 & 1.741 & 119054.0 & 122854.0 & 126375.0 & 130436.0 \\
\hline mqap1041 & $10^{17}$ & 6.222 & 7.156 & 6.760 & 8.105 & 24245.1 & 24000.7 & 24245.8 & 23313.3 \\
\hline mqap1042 & $10^{18}$ & 2.199 & 2.075 & 2.245 & 2.076 & 24114.9 & 25092.1 & 24736.9 & 24993.1 \\
\hline mqap1043 & $10^{18}$ & 1.176 & 1.219 & 1.237 & 0.907 & 25709.3 & 27852.8 & 25633.6 & 27028.5 \\
\hline mqap2041 & $10^{19}$ & 5.097 & 5.072 & 5.675 & 4.902 & 63237.5 & 64407.7 & 58227.1 & 65164.5 \\
\hline mqap2042 & $10^{19}$ & 4.678 & 4.188 & 4.215 & 4.113 & 66012.0 & 66821.5 & 65626.4 & 69821.2 \\
\hline mqap2043 & $10^{19}$ & 3.892 & 4.026 & 3.932 & 4.219 & 73037.1 & 71079.1 & 73457.2 & 72150.1 \\
\hline mqap3041 & $10^{20}$ & 1.115 & 1.068 & 0.977 & 1.008 & 93269.0 & 92824.1 & 97945.0 & 92839.0 \\
\hline mqap3042 & $10^{20}$ & 1.754 & 2.153 & 1.821 & 1.838 & 103954.0 & 87682.1 & 98771.0 & 97665.7 \\
\hline mqap3043 & $10^{20}$ & 1.374 & 1.679 & 1.518 & 1.469 & 110804.0 & 100624.0 & 106420.0 & 107446.0 \\
\hline mqap4041 & $10^{20}$ & 8.382 & 7.547 & 8.589 & 9.211 & 166838.0 & 161537.0 & 152980.0 & 155191.0 \\
\hline mqap4042 & $10^{20}$ & 4.342 & 6.292 & 4.596 & 5.195 & 156363.0 & 138090.0 & 152010.0 & 153548.0 \\
\hline mqap4043 & $10^{20}$ & 6.868 & 7.325 & 7.517 & 7.529 & 157845.0 & 144300.0 & 138431.0 & 157106.0 \\
\hline
\end{tabular}

\subsubsection{MQAP}

Results of PAES $\rightarrow$ mQAP are presented in Table 13. From the 36 instances, the recycling techniques presented better hypervolume and $\varepsilon$-add values for 26 and 31 instances, respectively. Statistical differences were pointed out by the KW test for 3 instances: 2022, 3023 and 2031. The verif method was outperformed by the clean method on the 2022 instance. The crowdd method outperformed the clean method on the
3023 and 2031 instances. The average time results were 2.01, $1.98,1.98$ and 2.04 for the clean, random, crowdd and verif methods, respectively.

\subsubsection{Continuous Problems}

Table 14 shows the results obtained by PAES and its variants. WFG1 hypervolumes have to be multiplied by $10^{-5}$.

No statistical difference was verified for the DTLZ1 ${ }^{2}$ and DTLZ2 $^{2}$ instances. For $d=3$ and $d=4$, the crowdd method 
Table 14. Results of the PAES variants for the continuous problems

\begin{tabular}{|c|c|c|c|c|c|c|c|c|}
\hline Problem & \multicolumn{4}{|c|}{ Hypervolume } & \multicolumn{4}{|c|}{$\varepsilon-a d d$} \\
\hline & clean & crowdd & random & verif & clean & crowdd & random & verif \\
\hline DTLZ1 $^{2}$ & 0.045 & 0.043 & 0.042 & 0.045 & 0.1100 & 0.1106 & 0.1308 & 0.1058 \\
\hline DTLZ1 $^{3}$ & 0.038 & 0.049 & 0.042 & 0.039 & 0.2067 & 0.1988 & 0.2204 & 0.2078 \\
\hline DTLZ1 $^{4}$ & 0.702 & 0.803 & 0.706 & 0.701 & 0.1999 & 0.1884 & 0.2083 & 0.2001 \\
\hline DTLZ2 $^{2}$ & 0.105 & 0.088 & 0.089 & 0.105 & 0.1331 & 0.1356 & 0.2323 & 0.1315 \\
\hline DTLZ2 $^{3}$ & 0.054 & 0.079 & 0.063 & 0.053 & 0.3547 & 0.3448 & 0.3342 & 0.3564 \\
\hline DTLZ2 $^{4}$ & 0.027 & 0.044 & 0.034 & 0.026 & 0.3673 & 0.3392 & 0.3384 & 0.3706 \\
\hline DTLZ3 $^{2}$ & 0.016 & 0.016 & 0.016 & 0.016 & 1.0030 & 1.0034 & 1.0179 & 1.0030 \\
\hline DTLZ3 $^{3}$ & 0.023 & 0.023 & 0.022 & 0.022 & 0.9072 & 0.9019 & 0.9090 & 0.9494 \\
\hline DTLZ3 $^{4}$ & 0.436 & 0.443 & 0.410 & 0.426 & 0.8663 & 0.8169 & 0.8457 & 1.0512 \\
\hline DTLZ4 $^{2}$ & 0.0000 & 0.0000 & 0.0000 & 0.0000 & 1.027 & 1.027 & 1.027 & 1.027 \\
\hline DTLZ4 $^{3}$ & 0.000 & 0.000 & 0.000 & 0.000 & 0.8253 & 0.8253 & 0.8531 & 0.8194 \\
\hline DTLZ4 $^{4}$ & 0.000 & 0.000 & 0.000 & 0.000 & 0.9364 & 0.9364 & 0.9399 & 0.9425 \\
\hline DTLZ5 $^{2}$ & 0.107 & 0.090 & 0.095 & 0.107 & 0.1331 & 0.1356 & 0.2136 & 0.1352 \\
\hline DTLZ5 $^{3}$ & 0.022 & 0.015 & 0.020 & 0.023 & 0.1876 & 0.1506 & 0.2469 & 0.1678 \\
\hline DTLZ5 $^{4}$ & 0.031 & 0.030 & 0.024 & 0.031 & 0.2206 & 0.1891 & 0.2779 & 0.2283 \\
\hline DTLZ6 $^{2}$ & 0.089 & 0.076 & 0.073 & 0.089 & 0.1255 & 0.1285 & 0.2241 & 0.1255 \\
\hline DTLZ6 $^{3}$ & 0.215 & 0.211 & 0.151 & 0.215 & 0.1330 & 0.1231 & 0.2483 & 0.1290 \\
\hline DTLZ6 $^{4}$ & 0.325 & 0.327 & 0.203 & 0.325 & 0.4402 & 0.4060 & 0.5132 & 0.4373 \\
\hline DTLZ7 $^{2}$ & 0.254 & 0.200 & 0.245 & 0.254 & 0.1093 & 0.1254 & 0.1725 & 0.1060 \\
\hline DTLZ7 $^{3}$ & 0.231 & 0.229 & 0.202 & 0.234 & 1.3656 & 1.1149 & 1.1828 & 1.3521 \\
\hline DTLZ7 $^{4}$ & 0.374 & 0.414 & 0.316 & 0.396 & 2.6058 & 2.0166 & 2.1985 & 2.5543 \\
\hline LZ09F1 & 0.410 & 0.397 & 0.381 & 0.410 & 0.3339 & 0.3333 & 0.3730 & 0.3361 \\
\hline LZ09F2 & 0.031 & 0.031 & 0.031 & 0.031 & 0.3538 & 0.3538 & 0.3604 & 0.3529 \\
\hline LZ09F3 & 0.097 & 0.097 & 0.093 & 0.097 & 0.2715 & 0.2715 & 0.2893 & 0.2752 \\
\hline LZ09F4 & 0.024 & 0.024 & 0.024 & 0.024 & 0.3129 & 0.3125 & 0.3280 & 0.3092 \\
\hline LZ09F5 & 0.066 & 0.066 & 0.066 & 0.066 & 0.2718 & 0.2717 & 0.2806 & 0.2656 \\
\hline LZ09F6 & 0.004 & 0.004 & 0.004 & 0.004 & 0.5035 & 0.4812 & 0.5077 & 0.4929 \\
\hline LZ09F7 & 0.016 & 0.016 & 0.016 & 0.016 & 0.3062 & 0.3062 & 0.3062 & 0.3191 \\
\hline LZ09F8 & 0.415 & 0.415 & 0.415 & 0.415 & 0.3583 & 0.3583 & 0.3583 & 0.3583 \\
\hline LZ09F9 & 0.126 & 0.126 & 0.126 & 0.126 & 0.3858 & 0.3857 & 0.3858 & 0.3860 \\
\hline WFG1 & 5.750 & 5.886 & 4.409 & 6.291 & 0.4180 & 0.4163 & 0.4759 & 0.4121 \\
\hline WFG2 & 10.213 & 12.226 & 10.101 & 10.338 & 2.1665 & 1.1385 & 1.8536 & 2.1372 \\
\hline WFG3 & 4.578 & 4.098 & 3.895 & 4.586 & 0.7232 & 0.6466 & 0.7726 & 0.7341 \\
\hline WFG4 & 5.796 & 11.726 & 8.025 & 6.030 & 4.5725 & 2.2579 & 3.4247 & 4.4687 \\
\hline WFG5 & 7.006 & 10.869 & 7.256 & 6.489 & 4.0115 & 2.0612 & 3.1523 & 4.2826 \\
\hline WFG6 & 5.405 & 7.149 & 4.925 & 5.492 & 2.7511 & 2.1947 & 2.4678 & 2.6503 \\
\hline WFG7 & 5.818 & 7.479 & 5.068 & 5.740 & 3.2204 & 2.9108 & 3.3369 & 3.2291 \\
\hline WFG8 & 9.465 & 11.301 & 9.144 & 9.932 & 2.4381 & 2.2345 & 2.4002 & 2.3688 \\
\hline WFG9 & 4.911 & 7.677 & 6.321 & 4.750 & 4.1911 & 3.0298 & 3.9466 & 4.4855 \\
\hline Fonseca & 0.268 & 0.238 & 0.202 & 0.270 & 0.1416 & 0.1808 & 0.4446 & 0.1505 \\
\hline Kursawe & 185.293 & 185.375 & 177.479 & 186.269 & 1.2915 & 1.3283 & 2.6610 & 1.3345 \\
\hline Viennet 2 & 275.147 & 272.548 & 267.882 & 275.257 & 0.0317 & 0.2355 & 0.0758 & 0.0306 \\
\hline Viennet3 & 2.929 & 2.367 & 2.931 & 2.878 & 0.4331 & 0.7484 & 0.8568 & 0.4536 \\
\hline
\end{tabular}

outperformed the others concerning the hypervolume indicator. The statistical test did not show significant differences for the LZ09 functions. The crowdd method outperformed the other methods concerning both quality indicators on the WFG2, WFG4, WFG5 and WFG6 instances. It also outperformed the other methods concerning the hypervolume indicator on WFG7 and WFG9 instances. The clean and verif methods were the best ones for the Fonseca, Kursawe, Viennet 2 and Viennet 3 problems. The average processing times of the clean, crowdd, random and verif methods were 1.66s, $1.87 \mathrm{~s}, 1.85 \mathrm{~s}$ and $1.94 \mathrm{~s}$, respectively.

\subsubsection{Discussion about the results of the PAES variants}

The recycling techniques were less efficient for PAES than for the other optimizers. This fact may have occurred due to the nature of the algorithm which maintains a single solution in its population. The recycling methods only interfered on the
PAES archive, but the guide solution was not changed. For the previous algorithms, the recycling techniques interfered on the population.

\section{Conclusions and Future Works}

This study investigated methods to recycle nondominated solutions discarded by multi-objective optimizers. This investigation was motivated by the conclusions of previous studies presented by [8] and [9] which concluded that most filtering techniques used by multi-objective algorithms might deteriorate (or present other significant problems). Thus, this paper presented methods to prevent deterioration and improve the quality of the approximation sets generated by multiobjective optimizers. To accomplish this task, an archive of discarded solutions (recycling bin) was included to each optimizer. Three distinct techniques were proposed to man- 
age the solutions of the recycling bin. The techniques were called random, crowdd and verif. Periodically solutions of the recycling bin were scanned to be inserted back to the optimizer. The recycling techniques were implemented into three known multi-objective algorithms: NSGA-II, SPEA2 and PAES. Computational experiments with 91 instances of discrete and continuous problems were done. The results showed that the capacity of the recycling techniques to lessen deterioration effects depended on the optimizer and the problem.

Based on the results of the experiments, the SPEA2 was the most benefited algorithm by the recycling techniques. The clean version performed poorly in comparison to the recycling variants. The least benefited algorithm was the PAES. It occurred since the recycling techniques did not directly interfere on the guiding solution. The recycling techniques only acted on the external archive. The best recycling technique for the NSGA-II was the periodical verification of deterioration (verif method). The recycling technique based on crowding distance (crowdd method) brought the best benefit for the PAES. A noteworthy fact is that the crowding distance did not present good results on the experiments for fixed input sequences, especially when those sequences were taken from problems with more than two objectives.

Future works will investigate other low complexity metrics to guide the recycling methods, such as those proposed by [30]. As at least one recycling method was able to improve the optimizers, future works will also investigate hybrid recycling methods and extend this study to many-objective problems.

\section{Author contributions}

All authors discussed the methods of recycling solutions on multi-objective optimizers and planned the experiments. H. G. de Medeiros implemented the algorithms and ran the experiments. E. F. G. Goldbarg and M. C. Goldbarg supervised the whole work. All authors analyzed the results and contributed to the final manuscript.

\section{References}

[1] ZITZLER, E.; LAUMANNS, M.; THIELE, L. SPEA2: Improving the strength pareto evolutionary algorithm. Evol. Methods Des. Optim. Control, v. 1, n. 1, p. 95-100, 2002.

[2] KNOWLES, J.; CORNE, D. The pareto archived evolution strategy: a new baseline algorithm for pareto multiobjective optimisation. In: Evolutionary Computation, 1999. CEC 99. Proceedings of the 1999 Congress on. Washington, USA: IEEE, 1999. (CEC, '99).

[3] ZITZLER ECKARTAND KÜNZLI, S. Indicator-based selection in multiobjective search. In: Parallel Problem

Solving from Nature - PPSN VIII. Berlin, Heidelberg:

Springer Berlin Heidelberg, 2004. (LNCS, v. 3242).

[4] KNOWLES, J.; CORNE, D. Properties of an adaptive archiving algorithm for storing nondominated vectors. Trans. Evol. Comp, v. 7, n. 2, p. 100-116, 2003.
[5] KNOWLES, J. D. Local-Search and Hybrid Evolutionary Algorithms for Pareto Optimization. Tese (Doutorado) - University of Reading, UK, Reading, UK, 2002.

[6] LAUMANNS, M. et al. On the Convergence and Diversity-Preservation Properties of Multi-Objective Evolutionary Algorithms. 2001.

[7] KNOWLES JOSHUAAND CORNE, D. Bounded pareto archiving: Theory and practice. In: GANDIBLEUX, X. et al. (Ed.). Metaheuristics for Multiobjective Optimisation. Berlin, Heidelberg: Springer Berlin Heidelberg, 2004. (Lecture Notes in Economics and Mathematical Systems, v. 535).

[8] LÓPEZ-IBÁÑEZ, M.; KNOWLES, J. D.; LAUMANNS, M. On sequential online archiving of objective vectors. In: TAKAHASHI, R. H. C. et al. (Ed.). Evolutionary Multi-criterion Optimization (EMO 2011), Lecture Notes in Computer Science. Berlin, Germany: Springer, 2011. (LNCS, v. 6576).

[9] MEDEIROS, H. G. de; GOLDBARG, E. F. G.; GOLDBARG, M. C. Analyzing limited size archivers of multi-objective optimizers. In: Brazilian Conference on Intelligent Systems. São Paulo, Brazil: IEEE, 2014. (BRACIS, '14).

[10] DEB, K. et al. A fast and elitist multiobjective genetic algorithm: NSGA-II. IEEE Trans. Evol. Comput., v. 6, n. 2, p. 182-197, 2002.

[11] LAUMANNS, M.; ZENKLUSEN, R. Stochastic convergence of random search methods to fixed size pareto front approximations. Eur. J. Oper. Res., v. 213, n. 2, p. 414-442, 2011.

[12] POZO, A.; BRITTO, A. B. de. Using archiving methods to control convergence and diversity for many-objective problems in particle swarm optimization. In: ABBASS, H.; ESSAM, D.; SARKER, R. (Ed.). WCCI 2012 IEEE World Congress on Computational Intelligence. Brisbane, Australia: IEEE, 2012. (CEC,' 12).

[13] JIN, H.; WONG, M.-L. Adaptive, convergent, and diversified archiving strategy for multiobjective evolutionary algorithms. Expert Syst. Appl., v. 37, n. 12, p. 8462-8470, 2010.

[14] HANNE, T. On the convergence of multiobjective evolutionary algorithms. Eur. J. Oper. Res., v. 117, n. 3, p. 553-564, 1999.

[15] BRINGMANN, K.; FRIEDRICH, T. Don't be greedy when calculating hypervolume contributions. In: GARIBAY, I. et al. (Ed.). Proceedings of the Tenth ACM SIGEVO Workshop on Foundations of Genetic Algorithms. Florida, USA: ACM, 2009. (FOGA, '09).

[16] ZITZLER, E.; THIELE, L. A comparative case study and the strength pareto approach. IEEE Trans. Evol. Comput., v. 3, n. 4, p. 257-271, 1999. 
[17] ZITZLER, E. et al. Performance assessment of multiobjective optimizers: an analysis and review. IEEE Trans. Evol. Comput., v. 7, n. 2, p. 117-132, 2003.

[18] DURILLO, J. J.; NEBRO, A. J. jmetal: A java framework for multi-objective optimization. Adv. Eng. Softw., v. 42 , n. 10 , p. $760-771,2011$.

[19] KRUSKAL, W. H.; WALLIS, W. A. Use of ranks in one-criterion variance analysis. J. Amer. Statist. Assoc., v. 47, n. 260, p. 583-621, 1952.

[20] BLEULER, S. et al. PISA - a platform and programming language independent interface for search

algorithms. In: FONSECA, C. M. et al. (Ed.). Evolutionary Multi-Criterion Optimization (EMO 2003). Berlin: Springer, 2003. (Lecture Notes in Computer Science, v. 2632).

[21] KOOPMANS, T. C.; BECKMANN, M. Assignment problems and the location of economic activities.

Econometrica, v. 25, n. 1, p. 53-76, 1957.

[22] KNOWLES, J.; CORNE, D. Instance generators and test suites for the multiobjective quadratic assignment problem. In: FONSECA, C. et al. (Ed.). Evolutionary Multi-Criterion Optimization, Second International Conference, EMO 2003. Faro, Portugal: Springer, 2003. (LNCS, v. 2632).

[23] HUBAND, S. et al. A scalable multi-objective test problem toolkit. In: COELLO, C. C.; AGUIRRE, A. H.; ZITZLER, E. (Ed.). Evolutionary Multi-Criterion Optimization. 1. ed. Berlin, Germany: Springer Berlin Heidelberg, 2005, (Lecture Notes in Computer Science, v. 3410). p. 280-295.
[24] LI, H.; ZHANG, Q. Multiobjective optimization problems with complicated pareto sets, MOEA/D and NSGA-II. IEEE Trans, Evol. Comput., v. 13, n. 2, p. 284-302, 2009.

[25] DEB, K. et al. Scalable multi-objective optimization test problems. In: Congress on Evolutionary Computation. Hawaii, USA: IEEE Press, 2002. (CEC, '02).

[26] ZITZLER, E.; DEB, K.; THIELE, L. Comparison of multiobjective evolutionary algorithms: Empirical results.

[27] VIENNET, R.; FONTEIX, C.; MARC, I. Multicriteria optimization using a genetic algorithm for determining a pareto set.

[28] FONSECA, C. M.; FLEMING, P. J. An overview of evolutionary algorithms in multiobjective optimization. Evol. Comput., v. 3, n. 1, p. 1-16, 1995.

[29] KURSAWE, F. A variant of evolution strategies for vector optimization. In: SCHWEFEL, H.-P.; MäNNER, R. (Ed.). Parallel Problem Solving from Nature. 1st Workshop, PPSN I, volume 496 of Lecture Notes in Computer Science. Berlin, Germany: Springer-Verlag, 1991. (PPSN, '91).

[30] KÖPPEN, M.; YOSHIDA, K. Substitute distance assignments in nsga-ii for handling many-objective optimization problems. In: Proceedings of the 4th International Conference on Evolutionary Multi-criterion Optimization. Berlin, Heidelberg: Springer-Verlag, 2007. (EMO, '07). 